\title{
De la ruche-tronc à la ruche à cadres : ethnoécologie historique de l'apiculture en Cévennes
}

From log hive to frame hive: ethnoecological history of beekeeping in Cevennes

Ameline Lehébel-Péron, Daniel Travier, Alain Renaux, Edmond Dounias et Bertrand Schatz

\section{(2) OpenEdition}

Journals

Édition électronique

URL : http://journals.openedition.org/ethnoecologie/2531

DOI : 10.4000/ethnoecologie.2531

ISSN : 2267-2419

Éditeur

Laboratoire Eco-anthropologie et Ethnobiologie

Référence électronique

Ameline Lehébel-Péron, Daniel Travier, Alain Renaux, Edmond Dounias et Bertrand Schatz, « De la ruche-tronc à la ruche à cadres : ethnoécologie historique de l'apiculture en Cévennes », Revue d'ethnoécologie [En ligne], 9 | 2016, mis en ligne le 01 juillet 2016, consulté le 30 avril 2019. URL : http:// journals.openedition.org/ethnoecologie/2531 ; DOI : 10.4000/ethnoecologie.2531

Ce document a été généré automatiquement le 30 avril 2019

\section{cc) $(1) \odot$}

Revue d'ethnoécologie est mis à disposition selon les termes de la licence Creative Commons Attribution - Pas d'Utilisation Commerciale - Pas de Modification 4.0 International. 


\title{
De la ruche-tronc à la ruche à cadres : ethnoécologie historique de l'apiculture en Cévennes
}

From log hive to frame hive: ethnoecological history of beekeeping in Cevennes

\author{
Ameline Lehébel-Péron, Daniel Travier, Alain Renaux, Edmond Dounias et \\ Bertrand Schatz
}

\section{Introduction}

L'apiculture est une production alimentaire ubiquiste et très ancienne. Les exemples des cueilleurs de miel, que l'on retrouve en Afrique, en Asie, en Amérique et, dans une moindre mesure, en Europe, sont le signe d'une grande ancienneté des usages humains du miel (Barrau 1983, Crane 1999). Historiquement, le miel est longtemps resté la source principale de sucre car la plus disponible in natura sur la planète (Crittenden 2011, Dounias et al. 2011), avant d'être progressivement remplacée durant la révolution industrielle du XIX ${ }^{e}$ et $\mathrm{XX}^{\mathrm{e}}$ siècle en Europe par le sucre de betterave (Crane 1999). Plus développée en Europe de l'Est qu'en Europe de l'Ouest, l'apicollecte (collecte des rayons de miel dans les colonies sauvages) a été rapidement supplantée par l'adoption de ruches conçues à partir de matériaux locaux : bois, liège, paille... qui ont façonné la diversité des pratiques et des paysages apicoles à travers le Vieux Continent (Marchenay 1979, Crane 1999). À partir du xx ${ }^{\mathrm{e}}$ siècle, c'est la ruche à cadres qui devient partout la plus utilisée car elle permet de meilleurs rendements en miel et la transhumance vers des sources mellifères saisonnières. Ce changement de ruche et de mobilité bouleverse profondément les pratiques apicoles par l'adoption de nouveaux outils (extracteur, vêtements de protection...), l'élevage de nouvelles races d'abeilles et d'hybrides (italienne, caucasienne, carniolienne l'hybride Buckfast...) et la valorisation d'autres produits de la ruche (propolis, pollen, gelée royale). Plus récemment, la diffusion rapide de l'acarien Varroa destructor et la propagation plus localisée de différents agents pathogènes (virus, 
bactéries, champignons, etc.) obligent les apiculteurs à réaliser des traitements sanitaires afin de limiter les mortalités parfois importantes. Cette trame désormais bien connue de l'évolution de l'apiculture est la même partout en Europe, avec des variantes sur l'occurrence de ses différents épisodes. Une conséquence dommageable de cette évolution commune est une inclination à en occulter les circonstances locales ou régionales particulières et les répercussions en matière de conservation et de développement.

Des siècles durant, l'apiculture en Cévennes, tout comme ailleurs en Europe, s'est inscrite dans le paysage (Maire \& Laffly 2015), s'appuyant sur des matériaux du cru (pierres, végétaux, etc.) et l'amélioration empirique de l'expertise locale, afin de mieux répondre aux besoins domestiques des paysans (production alimentaire et médicinale) et à leur solvabilité (substituts de monnaie, règlement d'impôts, prestations sociales dans le cadre d'échange, de dots ou d'héritages). L'apiculture était vectrice d'un véritable système socio-écologique dont les fondements environnementaux et sociaux sont propres à chaque région. C'est l'ensemble de ce système qui a été transformé au début du xxe siècle par l'arrivée des ruches à cadres mais aussi par le contexte économique et social remodelé par la révolution industrielle. Cette transformation est rarement prise en considération par les divers acteurs locaux (collectivités, société civile, agences de conservation, opérateurs économiques) en charge de la gestion territoriale. Il est pourtant désormais reconnu que la négligence des faits historiques est souvent à l'origine d'erreurs et de mauvaises interprétations dans l'établissement de stratégies de conservation et de restauration (Swetnam et al. 1999, Egan \& Howell 2005). L'approche issue de l'écologie historique est étendue ici à l'ethnoécologie, ce qui revient à considérer en détail les interactions biologiques et socioculturelles qui ont tissé l'histoire d'un système apicole combinant intimement l'écologie de l'abeille et de la ruche, les conditions environnementales de cet élevage, les savoir-faire des apiculteurs et les contextes économique et historique.

3 L'étude de l'histoire de l'apiculture que nous présentons concerne la région des Cévennes, dont les limites sont définies par celles du territoire d'action du Parc national des Cévennes (PnC). Dans son ouvrage Histoire des Cévennes, Patrick Cabanel (2009) déplore à juste titre l'absence de connaissances précises sur les époques anciennes de l'histoire cévenole. Cependant, les ruches-troncs alignées en terrasses sont caractéristiques de l'apiculture dite traditionnelle dans cette région (Figure 1) qui compte parmi la mieux conservée de France (Bertrand 2016). Reconnues depuis longtemps comme une «terre à miel », les Cévennes voient leurs paysages modelés par ces ruchers dont l'élément structurant est la ruche-tronc : celle-ci consiste en un tronc de châtaignier, évidé et fermé d'un couvercle du même bois puis recouvert d'une lauze de schiste largement débordante (Figure 1).

4 L'étude des ruches-troncs que nous restituons ici a été motivée par la double volonté du PnC de 1) documenter le patrimoine naturel et culturel lié à l'apiculture dans un objectif de gestion conservatoire ainsi que de 2) favoriser cette filière agricole dans sa diversité en prenant en compte son évolution historique. La recherche conduite s'est appuyée sur la compilation de documents d'archives, d'articles scientifiques et de témoignages, pour tenter de répondre à trois questions interdépendantes: 1) Quels types de ruches ont marqué de leur empreinte les paysages cévenols au fil de cette histoire 2) Quel a été le déroulé historique des races d'abeilles et des pratiques apicoles en Cévennes? 3) Quels ont été les divers produits de la ruche valorisés dans cette région? Notre objectif global 
est de reconstituer de façon détaillée l'histoire ethnoécologique de l'apiculture en Cévennes.

Figure : 1 : Une partie des 365 ruches du grand rucher-tronc au site « Les Balmelles " près de Villefort

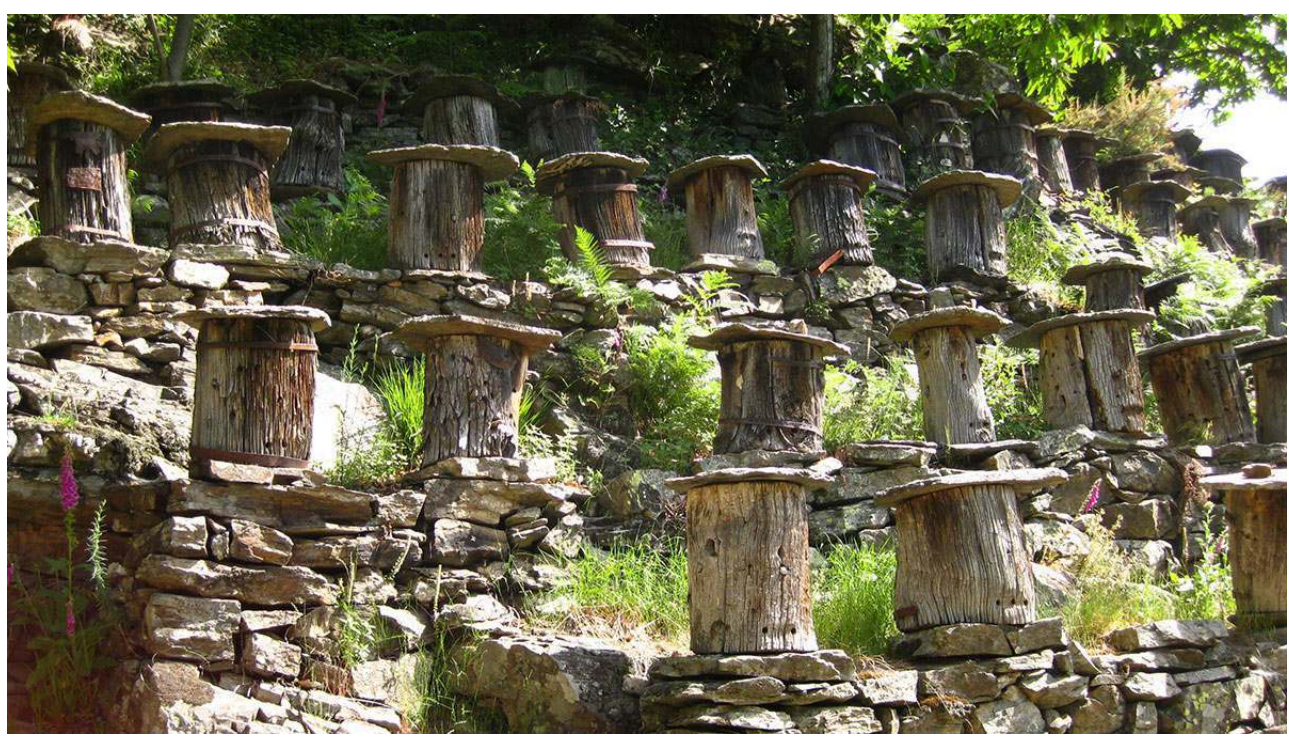

(c) A. Lehébel-Péron

\section{Matériel et méthodes}

5 Notre étude historique de l'apiculture cévenole a été menée sur le territoire du PnC (3 720 $\mathrm{km}^{2}$ ) situé au sud du massif Central et comptant plus de 150 communes et 73000 habitants (PnC 2007). Nous avons regroupé un ensemble d'informations permettant cette reconstitution historique en distinguant le contenant (ruche-tronc et ruche à cadres), le contenu (différentes races d'abeilles) et les produits (miel, cire et autres). Ces informations sont issues de documents d'archives, d'entretiens et de témoignages, ainsi que de publications scientifiques et de rapports d'experts non publiés. Les archives exploitées sont celles des deux départements constitutifs de la zone cœur du PnC. Elles sont disponibles sur demande aux Archives Départementales de la Lozère (ADL) ou à celles du Gard (ADG). Les pièces présentées ici portent des numéros du type $1 \mathrm{~N}, 2 \mathrm{ST}$, etc. qui permettent de retrouver leur cote d'archive (Lehébel-Péron 2014). Elles ont été enrichies par les archives personnelles de Daniel Travier (ethnohistorien et fondateur du Musée des vallées cévenoles) et d'Alain Renaux (ethnobotaniste en Cévennes). Des informations complémentaires, couvrant une période plus récente, ont été obtenues par observation participante durant trois années passées en Cévennes de 2011 à 2014 par Ameline Lehébel-Péron; elles consistent en des entretiens semi-directifs et des témoignages recueillis auprès de personnes ayant connu de leur vivant l'époque où les ruchers-troncs occupaient une place prépondérante dans l'économie domestique (Lehébel-Péron 2014). 


\title{
Résultats
}

\section{Déroulé historique du type de ruches utilisées en Cévennes}

\author{
De la Préhistoire au Moyen Âge : l'apparition de l'apiculture et le développement des \\ ruchers-troncs
}

\section{La Préhistoire}

6 Les plus anciens témoignages de la présence de l'homme sur le territoire de l'actuel PnC, contemporain de celui de Tautavel (environ 350000 ans BP) ont été trouvés dans les grottes et les avens du causse Méjean et ceux du causse de Sauveterre (Redon 1977). En Lozère, les premiers peuplements humains attestés datent du Paléolithique moyen (250 000 à 35000 ans BP) et sont plus marqués dans les basses Cévennes et sur les Causses qu'en Cévennes schisteuses (Redon 1977). Au Néolithique, les nombreuses traces de céramiques prouvent que toute la région des Cévennes était peuplée et les fouilles attestent d'une pratique de la transhumance ovine et de la chasse à proximité des massifs de l'Aigoual et du Lingas (Cabanel 2009). Les premiers villages sont édifiés sur les plateaux, souvent entourés de remparts rudimentaires, et plusieurs centaines de mégalithes, de menhirs et de dolmens confortent l'ancienneté de l'occupation humaine (PnC 2007). Dès 9000 ans BP, la transhumance de la plaine vers les pâturages plus verts des montagnes cévenoles serait effective, notamment pour les ovins ; il convient toutefois de préciser qu'une véritable transhumance n'aurait réellement débuté qu'à partir du Moyen Âge avec l'apparition des grands troupeaux dans les plus importantes abbayes (Vernhet A. et P. Coste comm. pers.). Les paysages sont essentiellement forestiers, couverts de chênaies et de hêtraies naturelles. En vieillissant, les arbres (plus particulièrement les châtaigniers) deviennent creux et offrent un habitat de choix pour les colonies d'abeilles mellifères alors exclusivement sauvages. Sans qu'aucune trace archéologique connue de poterie ayant contenu du miel, de la cire ou du pollen ne l'atteste, il est probable que, dès le Néolithique, dans les Cévennes comme ailleurs en Europe (Roffet-Salque et al. 2015), les humains aient récolté et consommé les produits (principalement le miel) issus de colonies d'abeilles sauvages (Marchenay 1979).

\section{L'Antiquité}

7 Malgré les invasions des Celtes puis celles des Romains pendant l'époque gallo-romaine, la population est présente partout en Cévennes où elle pratique intensément l'élevage. Elle se structure autour de plusieurs centres, reliés par des routes fréquentées entre hautes et basses Cévennes (Cabanel 2009) et le mont Lozère est déjà un haut-lieu de transhumance (Chevallier 1982). Comme pour la période préhistorique, peu d'indices archéologiques permettent de décrire l'apiculture ou l'apicollecte dans les Cévennes durant l'Antiquité. Le poète gallo-romain Sidoine Apollinaire, lors de ses voyages en Gaule méridionale (vraisemblablement entre 464 et 467) évoque la douceur méditerranéenne des habitations des basses Cévennes avec leurs jardins délicieux "pareils à ceux qui embellissent le sommet de l'Hybla, fertile en miel» (Chaix 1866, Cabanel 2009). À l'antiquité, l'Hylba est un sommet de Sicile, connu pour son miel issu des ruches tressées en paille de férule (Ferula sp.) (Crane 1999). La comparaison du «sommet de l'Hybla » et des vallées cévenoles suggère qu'il existait, dès le $v^{e}$ siècle, des ruches en Cévennes. Nous 
ne possédons pas d'éléments suffisants pour connaitre ni le type de ruche ni le matériel de fabrication. Les colonies d'abeilles sauvages s'installent spontanément dans des cavités d'arbres, mais à cette époque le châtaignier est encore une essence rare. En effet, les palynologues estiment que les traces les plus anciennes de la présence du châtaignier dans le Massif Central, et ses régions voisines datent de l'époque romaine ; l'expansion de la châtaigneraie ne débutera vraiment qu'au Moyen Âge (Travier 2006). Ainsi la ruche en tronc de châtaignier ne devait probablement pas encore être répandue à l'époque romaine, en revanche les ruches en paille ont pu être utilisées car les techniques de vannerie spiralée sont connues dès le Néolithique (Crane 1999).

\section{Le Moyen Âge}

Pendant cette période, aucune mention claire d'utilisation de ruche-tronc n'a pu être relevée et aucune source ne permet de connaître précisément les matériaux dans lesquels les ruches étaient construites. Cependant, un faisceau de présomptions permet d'apporter quelques indications sur l'apiculture cévenole moyenâgeuse. Les ruches pouvaient être construites en paille, en planches de bois ou creusées dans un tronc (Olivier de Serres 1600), ou même en pierre sèche: une ruche en pierre sèche est décrite en 1691 à Jouvenargues, en vallée longue dans le Gard (P. Rolland comm. pers). Il existe en Cévennes quelques traces de ruches-placard (construites en planches débitées) mais pas suffisamment pour témoigner d'une apiculture très développée à cette époque. Sans doute plus communes, les ruches en paille pourraient probablement s'être répandues dès l'époque médiévale dans le nord de la Lozère, en Margeride et en Gévaudan (D. Travier comm. pers., Brinkmann 1938, Crane 1999). Avant le refroidissement du XIV siècle, les productions cévenoles sont essentiellement méditerranéennes : olivier, vigne et céréales dont le seigle à paille longue très propice à la vannerie. Toutefois les termes vernaculaires bourgnion, brougnion, bornhon, bronhon qui sont les plus utilisés aux XVII et XVIII siècles pour désigner les ruches, ne permettent pas d'affirmer qu'il s'agisse de vanneries (D. Travier comm. pers., Marchenay 1979, Lehébel-Péron 2014). La forme de cylindre creux est à l'origine du nom bornhon décliné en bòrnha signifie "cavité ", "tronc creux». Le verbe bornhejar signifie "faire une ruche», "creuser une ruche». S'il s'agissait d'une fabrication en vannerie c'est le terme bridolar qui aurait été employé. Il est donc plausible d'envisager pour cette période l'introduction dans un récipient en vannerie d'un essaim prélevé in natura, dans une cavité ligneuse ou une anfractuosité rocheuse, pour en pratiquer l'élevage. Cependant, le nom même des ruches indique qu'aux Temps Modernes (1453-1789), elles sont essentiellement creusées dans des troncs de châtaignier.

9 Un travail de Jacques Galzin (1986) sur les toponymes des Cévennes gardoise et lozérienne, permet de déduire que de nombreuses plantations de châtaigniers ont été réalisées avant les années 1000 et 1100 sur des terrains peu boisés ou déboisés à cet effet. La châtaigne, en tant qu'unité d'échange, prend une part importante dans les redevances féodales (Travier 2006). Le bois de châtaignier se décomposant depuis le cœur, il est facile d'imaginer qu'à partir de l'observation de vieux châtaigniers creux, pouvant héberger des colonies d'abeilles, les gens du pays aient confectionné les premières ruches (Figure 2). Depuis le haut Moyen Âge, la forêt était ouverte à tous dans le contexte d'une économie essentiellement rurale. Cependant, à partir des années 1200, le statut de la forêt et des produits qu'on en tirait change radicalement. Au XIII ${ }^{\mathrm{e}}$ siècle, le développement urbain, et le commerce du bois de construction qui l'accompagne, incitent les seigneurs à se 
réserver l'usage exclusif de la forêt et de ses produits (Duby 1970). Faral (1948) rapporte l'établissement au niveau national des bigres sous Louis IX (qui règne de 1226 à 1270) : il s'agissait d'agents forestiers chargés de rechercher directement en forêt les essaims, de les élever et de recueillir le miel et la cire pour le compte d'un seigneur (Figure 2). Cependant, aucune source d'information n'atteste la présence de bigres dans les seigneuries cévenoles.

Bien que les informations sur le type de ruches utilisées fassent défaut, les redevances féodales gardoises, utilisant des unités de cire (1353) et de miel (1373) en guise de monnaie, confirment que l'apiculture se pratiquait en Cévennes au Moyen Âge (voir plus loin concernant les produits de la ruche). L'utilisation de produits de la ruche comme monnaie suggère fortement l'existence d'une production régulière, et soutient l'idée de l'existence d'une apiculture maitrisée plutôt que d'une apicollecte ponctuelle. Il faut attendre un acte de 1488 pour divulguer une information intéressante relative sur l'emplacement de cette apiculture. Le cens exigé (redevance en nature) est d'un carteron de miel pour une parcelle désignée comme Ranc de Label (Terrier de la Boissonade, archive consultable au «Lien du Chercheur Cévenol »: www.cevenols.fr); ce toponyme est interprété comme le ranc de l'Abel c'est-à-dire le ranc appartenant à Abel (ou de l'abeille qui se dit abelha en occitan). En langue occitane, le ranc désigne une roche escarpée, une paroi rocheuse qui constitue un emplacement propice à l'installation de ruches, la paroi faisant office d'abri. Il s'agit là d'une des premières mentions précises d'un élevage d'abeilles sur une succession de terrasses cévenoles, sans qu'il soit toutefois permis d'en déterminer le type de ruche (paille ou ruche-tronc).

11 Il faut en outre considérer qu'une ruche-tronc doit être couverte pour assurer sa protection contre la pluie et la neige. Cette protection a surement consisté dans les premiers temps en un élément de poterie, puis en une lauze de schiste probablement à partir du XVII siècle. En effet, dans les basses vallées des Cévennes, les tuiles de terre cuite sont fabriquées et utilisées dès l'époque romaine. Les compoix, inventaires des biens des populations pour revoir la base de l'impôt, et surtout les arpentements plus détaillés établis vers 1550 pour le diocèse de Nîmes et d'Uzès, fournissent des descriptions très précises de l'habitat (D. Travier comm. pers.). À cette époque, exception faite des maisons fortes et des châteaux, la majorité des maisons, est pourvue de toits de chaume. L'utilisation des lauzes de schistes se démocratise à la fin $d u \mathrm{XVI}^{\mathrm{e}}$ siècle, puis surtout au XVII ${ }^{e}$ siècle. Elle est concomitante à l'expansion de la châtaigneraie et l'utilisation du châtaignier comme source de bois de charpente pour supporter les toits en lauzes de schistes. Cependant, l'utilisation des lauzes existe déjà un à deux siècles avant cette période; coûteuse, elle est toutefois restreinte aux résidences bourgeoises et aux châteaux.

12 En résumé, tous les éléments pour l'avènement d'une ruche-tronc en Cévennes ne sont réunis qu'au Moyen Âge: l'apiculture y est attestée depuis plusieurs siècles, les châtaigneraies existent depuis le $\mathrm{x}^{\mathrm{e}}$ siècle, les bigres sont présents depuis le $\mathrm{XIII}^{\mathrm{e}}$ siècle, miel et cire font office de monnaie depuis le milieu du XIV ${ }^{\mathrm{e}}$ siècle et sont produits sur des rancs depuis la fin du $\mathrm{Xv}^{\mathrm{e}}$ siècle; enfin l'exploitation des lauzes de schiste est attestée dès le $\mathrm{Xv}^{\text {e }}$ siècle. Ainsi, malgré l'absence de preuve directe, il est vraisemblable que l'apiculture cévenole en ruche-tronc soit apparue à la fin du Moyen Âge, au XVI ${ }^{e}$ siècle. 
Figure 2: Gravure de 1774 de Johann Georg Krünitz (1728-1796) montrant à la fois la collecte de miel dans les arbres et la confection de ruche-tronc

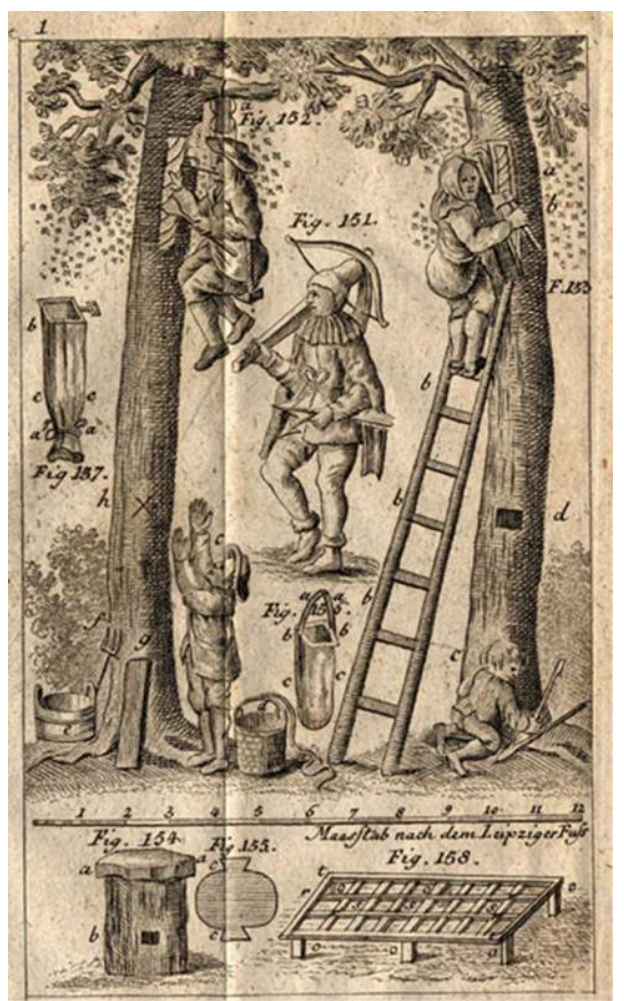

Cette gravure témoigne d'une apicollecte, qui se déroule en plusieurs étapes : il faut d'abord taper avec un bâton sur le tronc puis plaquer son oreille sur le tronc pour déterminer la présence d'abeilles (en bas à droite), puis tailler à la hache une porte dans le tronc, cette action semblant nécessiter le port d'une protection (en haut à droite). Ensuite, les rayons sont extraits à l'aide d'une sorte de spatule (en haut à gauche), chargés dans un sac faisant office de nacelle (milieu à gauche) pour être descendus puis stockés dans des vanneries ou des seaux en bois (en bas à gauche). Cette gravure illustre en outre comment ce prélèvement in natura a inspiré la confection des premières ruches-troncs (en bas à gauche), surement en châtaignier avec un couvercle construit en bois et une lauze placée par-dessus comme couvercle. Les personnages représentés sont surement les héritiers des sbires du XIIle siècle et porteurs des savoirs et des pratiques apicoles de l'époque.

(c) Encyclopédie de Krünig, www.kruenitz1.unitrier.de

\section{Du $X V I^{e}$ au $X V I I I^{e}$ siècle : expansion de la population, de la châtaigneraie et des ruches-troncs}

La forte augmentation démographique qui marque le $\mathrm{xVI}^{\mathrm{e}}$ siècle accompagne en Cévennes une extension sans précédent de la culture du châtaignier, un arbre qui fournit une nourriture abondante et régulière. Économiquement, l'abeille occupe déjà une place importante puisque le miel est alors l'unique source de sucre et que ses vertus médicinales sont également bien connues (D. Travier comm. pers.). C'est dans la deuxième moitié du XVI $\mathrm{e}^{\mathrm{e}}$ siècle que le bois de châtaignier devient suffisamment disponible pour être quasi systématiquement employé dans la fabrication des ruches.

Les premiers témoignages directs remontent au début du XVII ${ }^{\mathrm{e}}$ siècle, alors que la plupart des ruchers qui se construisent sont constitués de ruches en troncs de châtaignier couvertes d'une lauze. À cette époque, le châtaignier étant planté à outrance depuis 100 ans et utilisé dans toute l'économie domestique, il est certain que les brougnons de mouches à miel décrits dans les actes notariaux sont des ruches en troncs de 
châtaigniers ; ils seront par la suite appelés brus ou brusc, deux termes à rapprocher de l'occitan rusc pour nommer l'écorce selon Boissier de Sauvages (xvIII ${ }^{\mathrm{e}}$ siècle) et Mistral ( $\mathrm{XIX}^{\mathrm{e}}$ siècle) (D. Travier comm. pers.). Le notariat $\mathrm{du} \mathrm{Xv}^{\mathrm{e}}$ au XVIII ${ }^{\mathrm{e}}$ siècle illustre à la fois la mise en place de ces ruchers et l'importance du miel, de la cire et de l'arbre.

Ainsi, dans un acte de convention du 17 novembre 1627, il est question de deux personnes qui reconstruisent ensemble les murs pour enclore un rucher. L'apier est construit pour y mettre des ruches-troncs; les ruches et la récolte seront partagées équitablement entre ces deux personnes (Figure 3a). Dans une procédure du 19 mai 1694, sur la commune de l'Estréchure en vallée borgne, des personnes choisissent une pièce, c'est à dire une parcelle sous une falaise, une rancarède (Figure $3 b$ ). Dans ce document, où le terme rucher est utilisé à la place de ruche, l'homme demande à sa belle-sœur d'aller poser quelques ruches contre sa maison. Il développe ensuite le rucher et le ferme. Sa bellesœur lui en demande un loyer. Ce texte fait clairement allusion à l'élevage d'abeilles en ruches-troncs. Il convient de noter que le protagoniste n'est pas un agriculteur mais un artisan : l'apiculture s'instaure à la faveur d'une pluriactivité. Ces textes proviennent d'un travail de recherche de Daniel Travier sur tout le notariat de Saint-Jean-du-Gard, du Moyen Âge à 1789. Il ne fait guère de doute qu'en élargissant la prospection aux notariats de l'ensemble des Cévennes, beaucoup d'autres exemples seraient retrouvés concernant la mise en place de ruchers-troncs dont certains persistent encore aujourd'hui.

Figure 3 : Actes notariés du XVII ${ }^{\mathrm{e}}$ siècle liés à des activités apicoles avec des ruches-troncs

Convention - Acte du 17 novembre 1627 : « [...] méjarié et convention de brougnons de mouches à miel entre Messire Jean DUMAS marchand et David BOURDARIER cardeur lesquels ont fait ensemble et à communs frais un apié de brougnons de mouches à miel dans une pièce de terre appelé le Cade près d'une maison de ladite pièce sous les pactes que lesdits apié et brougnons demeurera méjier [à moitié] et tout le croit qui en parviendra sera divisé annuellement entre eux à moitié et tous despens sera à frais communs. Seront tenu de jour en jour et à réquisition de l'un d'entre eux entamer et clore de murailles à pierre sèche ledit apié et place de bourgnons à communs frais et en fin de méjerie de partager équitablement entre eux lesdits brougnons [...]»

ADG 2-58/369, notaire. (Archives D. Travier tome 3, p. 1539-1540)

Procédure - 19 mai 1694 : «[...] présente Isabeau DUGA veuve de David FRAISSINET du mas du Doudou paroisse de St-Martin-de-Corconac laquelle a exposé qu'il y a environ 9 ans que son beau-frère Jean MICHEL, maréchal à forge, du mas de la Paradine, paroisse de St-Martin-de-Corconac, lui aurait dit et prié de lui permettre de mettre quelques ruchers à une pièce rancarède [barre rocheuse] qu'elle a à côté de sa maison, disant que cela ne serait que pour un temps [...] ce que fit venir en sa maison Maitre Rousset notaire à Monteils et lui ayant dit et déclaré, comme elle lui avait donné la permission [...] ledit MICHEL en profita pour augmenter ses ruchers et même clore ladite pièce ce qu'elle n'aurait jamais voulu [...] aussi pour sa défense présente révoque solennellement susdite donation si jamais elle l'a fait [...] mais parait accepter d'engager ladite pièce pour 75 livres par an plus passage ou servitude de 25 livres pour s'y rendre [...] ».

ADG 2-58/495, notaire Paul Viala. (Archives D. Travier tome 7, p. 3489)

Selon D. Travier (comm. pers.), l'édification des ruchers est contemporaine à celle des habitations. L'habitat rural cévenol en hameaux est pratiquement implanté au XIII siècle. Il évoluera sur son site d'implantation avec l'accroissement démographique à partir du $\mathrm{XVI}^{\mathrm{e}}$ siècle. Cet habitat comprend l' habitation, les bâtiments d'exploitation et tous les aménagements à proximité : terrasses, aires de circulation, dispositifs d'écoulement du 
ruissellement, fontaine, aire à battre... et rucher à plus ou moins grande distance de l'habitation.

XIX siècle : peu de nouveaux ruchers en raison de la crise agricole et de l'exode rural

17 Dans les premières années du XIX ${ }^{e}$ siècle les infrastructures sont en place, la plupart des ruchers sont déjà implantés et le monde apicole cévenol ne connaît pas d'évolution notable jusqu'au milieu du siècle. C'est aussi pour les Cévennes un âge d'or lié au développement maximal de la sériciculture. Les crises agricoles et démographiques de la seconde moitié du xIX siècle vont fortement affecter l'apiculture des Cévennes. Crise démographique et exode rural sonnent le glas de l'implantation de nouvelles habitations ; les villages et les hameaux se dépeuplent progressivement ce qui réduit d'autant l'activité des terrasses de maraichage et de celles dédiées à l'apiculture, ces dernières étant plus étroites que pour les autres usages (D. Travier comm. pers.). Bâties par une main d'œuvre non spécialisée et saisonnière d'hiver, les terrasses à châtaigniers ne sont quasiment plus construites à partir des années 1840-1850; celles qui sont dédiées aux mûriers ne sont plus renouvelées du fait du ralentissement économique du commerce de la soie ; seules quelques terrasses vinicoles sont encore édifiées en Ardèche (Blanchemanche 1986). En Cévennes, surtout avant la seconde Guerre Mondiale, les premières ruches à cadres côtoient les ruches-troncs, sur les terrasses ou dans les ruchers des hameaux et de village (D. Travier comm. pers.). Ainsi la mise en place de nouveaux ruchers-troncs est plutôt rare à la fin du XIX ${ }^{\mathrm{e}}$ siècle même s'il reste possible de tout de même relever la création de petits ruchers comme c'est le cas par exemple de cet instituteur de Grisac, sur la commune du Pont-de-Montvert, qui met en place un rucher-troncs dans le jardin de l'école en 1874 (Figure 4). 
Figure 4 : Plan approximatif du jardin de l'école et du rucher de la maison et texte de description par l'instituteur de Grisac (commune du Pont de Montvert)

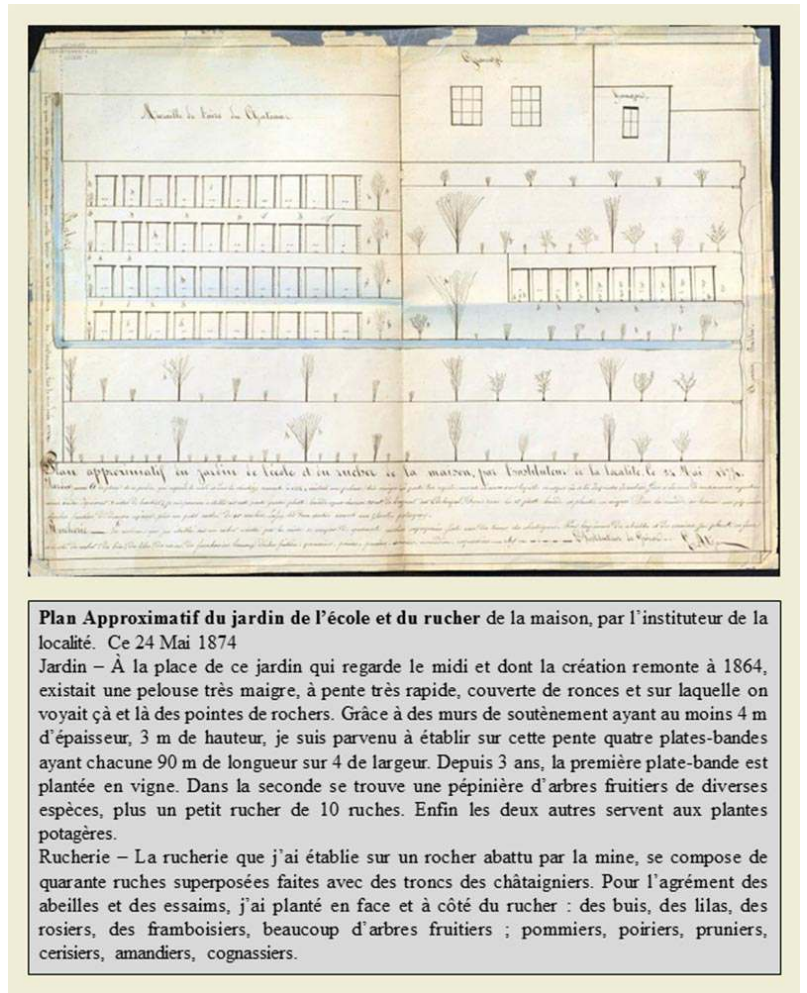

À noter dans le descriptif, le souci de l'orientation des terrasses, le nombre important de ruches attenantes à un établissement scolaire traduisant l'absence de crainte d'avoir autant d'abeilles à proximité des enfants, le détail de la nature d'une ruche-tronc, le souci de considérer à la fois les abeilles et les essaims et le bon sens de planter une diversité de plantes d'agrément et horticoles afin d'optimiser et de rendre compte de l'activité pollinisatrice des abeilles.

E. Atge en 1874 (CDRC (Centre Départemental deRessources Culturelles) :: 1 T 682-116)

\section{Fin $\mathrm{XIX}^{\mathrm{e}}$ siècle et début $\mathrm{XX}$ siècle : très lente diffusion des ruches à cadres}

En France, au début du $\mathrm{xx}^{\mathrm{e}}$ siècle, les techniques modernes d'apiculture, dites rationnelles, viennent progressivement supplanter une apiculture traditionnelle en ruches sédentaires bien implantées ${ }^{1}$. Les connaissances sur l'apiculture moderne se diffusent petit à petit dans tout le département de la Lozère. Dès 1862, le Bulletin de la société d'agriculture de la Lozère, vante l'apiculture rationnelle avec l'utilisation de la ruche à hausses (Figure 5haut). Trente ans plus tard, en 1893, cette même revue dénigre les « vieux procédés », expression utilisée en référence aux pratiques traditionnelles alors en vigueur (Figure 5bas) ; il est explicitement conseillé d'utiliser « la nouvelle ruche à cadres mobiles » (Figure 5bas). Les premières ruches Dadant (Figure 6) arrivent en France à partir de 1869 et deviennent les plus utilisées au début du $\mathrm{Xx}^{\mathrm{e}}$ siècle dans la plupart des régions (Crane 1999). 
Figure 5 : En haut, un apiculteur lorrain publie les principaux résultats du $2^{\text {ème }}$ congrès des apiculteurs français tenu les 16 et 17 août 1861 - En bas, texte de promotion de l'élevage des abeilles par l'emploi de la nouvelle ruche à cadres mobiles.

Quelle est la meilleure ruche ? Il résulte de la discussion [...] que les ruches à hausses et celles à chapiteau, notamment à la façon normande, sont celles qui presentent les plus d avantages pour la majorite des apiculteurs

L'élevage des abeilles. Par l'emploi de la nouvelle ruche à cadres mobiles. L'élevage des abeilles par l'adoption de nouvelles méthodes serait, pour de nombreux petits cultivateurs, pour toute personne possédant un jardin, une somme de revenus importants. Malgré le climat si favorable de notre pays, malgré sa flore si variée, on peut dire que l'apiculture est peu pratiquée en France. En Amérique, trente sociétés financières possédant chacune 5 à 6 millions de capitaux, emploient des sommes énormes à couvrir de ruches les terrains américains. Depuis longtemps, l'Italie expédie annuellement pour des millions de francs d'abeilles jaunes, sans contredit les plus fécondes et les plus actives. Tandis qu'en France, la plupart des cultivateurs en sont restés aux vieux procédés, aux anciennes habitations trop petites, défectueuses, où les colonies sont trop à l'étroit, manquant de place pour se développer, essaiment et s'émiettent au moment de la récolte la plus fructueuse, nos voisins les Américains ramassent des millions de tonnes de miel [...] et viennent nous faire concurrence sur nos marchés.

[...] Il faut une direction rationnelle, qu'elles aient toujours une nourriture suffisante, qu'elles soient logées dans des ruches dont la capacité puisse être augmentée ou diminuée selon les besoins, il faut que l'air à l'intérieur puisse être renouvelé mais [...] sans courant d'air [...]. Ces avantages ne se rencontrent pas dans la ruche en paille dont on fait encore en Normandie et en Bretagne un très grand usage [...] Cette méthode d'élevage des abeilles est la plus défectueuse que l'on connaisse. Aussi la nouvelle ruche à cadres mobiles employée avec un succès toujours croissant en Amérique et dans certaines contrées de l'Europe, ne saurait être trop recommandée dans nos départements.

Bulletin de la Société d'Agriculture du département de la Lozère, Tome 13, 1862

Bulletin de la Société d'agriculture, industrie, sciences et arts du département de la Lozère, Tome XLIV, 1893 (Noel 1893) 
Figure 6 : À gauche, la Ruche Dadant-Blatt présentée dans le Bulletin de la Société d'Agriculture du département de la Lozère, Tome XLVIII, 1896 (Conze 1896). À droite, la couverture du Petit traité de l'Apiculture moderne publié en Lozère en 1930 (Brousse 1930)

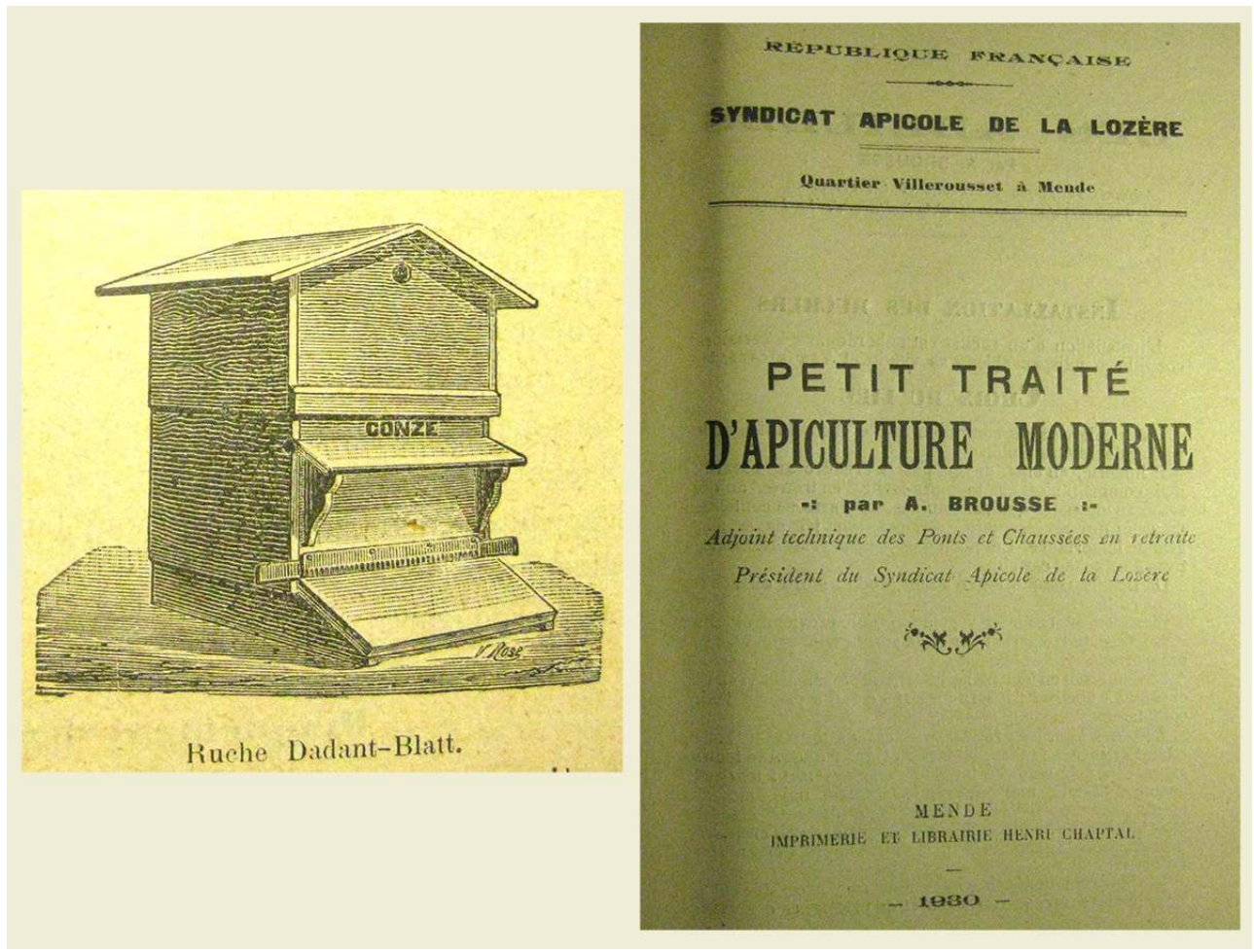

En Cévennes, malgré l'avènement de la ruche à cadres, l'apiculture traditionnelle en ruches-troncs qui a nourri la population cévenole depuis quelques siècles reste largement prédominante jusqu'au début $\mathrm{du} \mathrm{xx}^{\mathrm{e}}$ siècle. Les archives départementales de la Lozère conservent des monographies communales rédigées par des instituteurs dans leurs communes d'affectation à la demande de leur hiérarchie. À travers ces écrits qui commencent à mentionner l'élevage de reines, s'esquissent différentes intensités d'activité apicole. «Certains cultivateurs font un grand revenu des abeilles en ont jusqu'à 100 ruches, faites avec des troncs de châtaigniers ». Ces vastes ruchers qui font déjà penser à l'apiculture professionnelle actuelle, se déploient aux côtés d'une activité plus modeste et pluriactive, typiques d'une apiculture familiale en ruches-troncs où « chaque ferme cévenole a son rucher plus ou moins important, mais comptant en moyenne de 20 à 30 ruches " (extraits des monographies des communes de Saint-Andéol-de-Clerguemort et de Saint-Etienne-Vallée-Française, respectivement, rédigées entre 1914 et 1945, ADL 1M).

Dans la première moitié $\mathrm{du} \mathrm{xx}^{\mathrm{e}}$ siècle, malgré les efforts d'un monde apicole "savant " pour inciter à la modernité en apiculture, la ruche à cadres, pourtant connue, est encore peu utilisée dans les Cévennes. Dans les recensements communaux existants pour les communes lozériennes de Saint-Julien d'Arpaon et de Cassagnas, aucune ruche à cadres n'est répertoriée avant 1926 et 1936 respectivement. Il en va de même dans la commune de Fraissinet-de-Fourques qui ne possédait aucune ruche à cadres durant l'entre-deuxguerres alors que le cheptel apicole avoisinait les 2000 ruches-troncs, soit une moyenne de 10 à 30 ruches par famille (Clément 2015). Pourtant dès les années 1930, l'apiculture semble prendre une place de plus en plus importante dans la pluriactivité agricole. Le Syndicat Apicole de Lozère, créé le 24 juin 1923, est très favorable à une modernisation du 
cheptel (ADL 2W1134). Le désormais classique "Petit traité d'apiculture moderne» (Brousse 1930) dans lequel il est exclusivement question d'apiculture en ruches à cadres (Figure 6) est édité à Mende. Dans les expositions départementales d'apiculture, comme celle organisée en 1936 à Saint-Chely d'Apcher au nord de la Lozère, les modèles de ruches exposés et le matériel associé ne concernent que des ruches à cadres. Des formations sont même organisées par ces nouveaux syndicats pour présenter les nouvelles techniques et les nouveaux équipements : enfumoirs, protections, extracteurs, etc. (Clément 2015).

En 1943, l'administration agricole organise une des enquêtes de printemps et d'automne (ADL 4ST et ADL 5ST). Exceptionnellement, celle-ci recense également le nombre de ruches détenues par les paysans de chaque commune lozérienne et distingue les ruches ordinaires des ruches à cadres déclarées, donc en activité car exploitées par l'agriculteur déclarant (Figure 7a). Au total (surement sous-estimé), 2426 ruches-troncs sont déclarées pour 694 ruches à cadres, montrant ainsi qu'au milieu du $\mathrm{xx}^{\mathrm{e}}$ siècle, les ruches traditionnelles sont encore quatre fois plus nombreuses que les modernes. Les ruchestroncs traditionnelles, confectionnées à partir de matériaux directement prélevés alentour, sont plus simples à construire et à entretenir que les ruches à cadres, plus chères et plus compliquées à fabriquer. Les Cévenols ne sont pas riches et estiment que les troncs hérités de leurs ancêtres, emplis d'abeilles et de miel, ont encore de beaux jours devant eux! Les cartes de répartition communales des deux types de ruches établies d'après cette enquête montrent que l'activité apicole est plus développée au sud du département qu'au nord, quel que soit le type de ruche (Figure $7 b$ ). Une lecture plus détaillée fait ressortir que les "ruches ordinaires" restent très nombreuses dans les vallées cévenoles, tandis que les grands ruchers modernes de ruches à cadres se sont plutôt déployés dans le Causse Méjean, donc dans un paysage sans châtaignier où la ruche traditionnelle était confectionnée à base de paille ou d'osier.

Figure $7 \mathrm{a}$ : Formulaire du recensement d'automne 1943 ; bilan par canton pour l'arrondissement de Florac (ADL 4ST \& ADL 5ST)

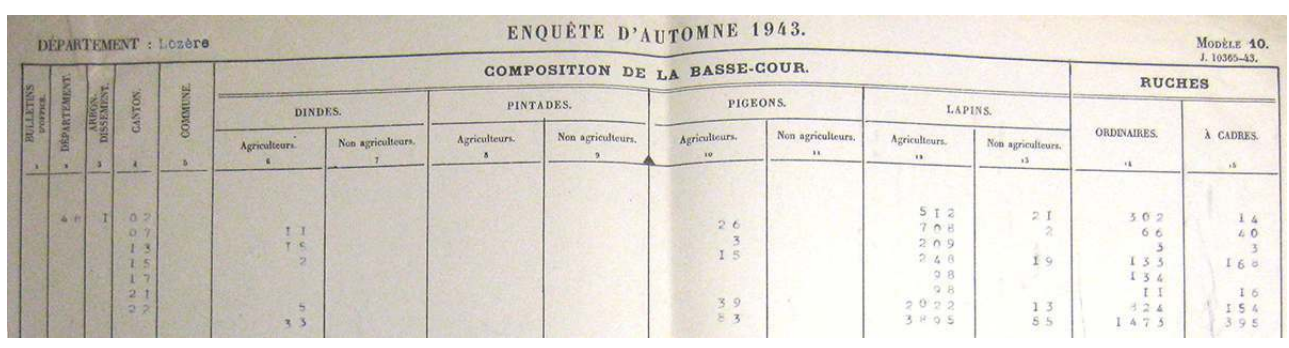


Figure $7 \mathrm{~b}$ : Cartes du nombre de ruches déclarées par commune pour le département de la Lozère réalisées à partir des enquêtes agricoles de 1943 (ADL 4ST et ADL 5ST)

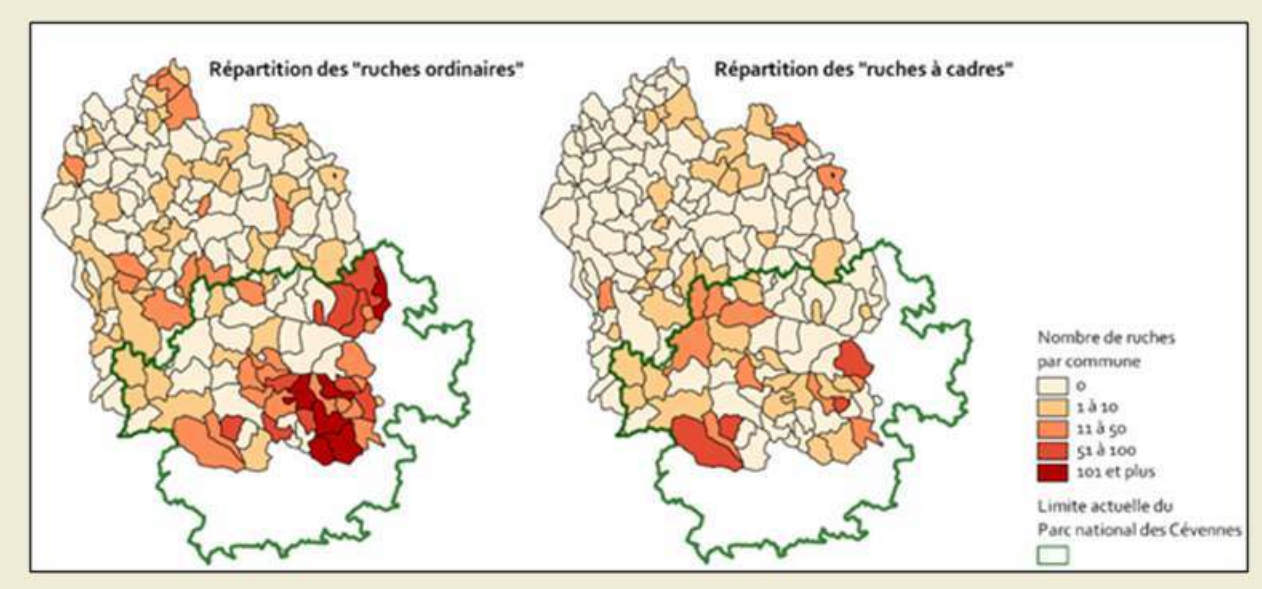

Dans un autre recensement datant également de 1943 et portant sur les 6 communes de Saint-Julien-d'Arpaon, Cassagnas, La-Salle-Prunet, Barre-des-Cévennes, Saint-André-de Lancize, et Saint-Privat-de-Vallongue, 379 ruches-troncs $(85,4 \%)$ sont déclarées pour 65 ruches à cadres (recensement s'adressant uniquement aux agriculteurs). En 1973, lors d'un recensement réalisé par un garde du PnC dans les mêmes communes, les chiffres révèlent la présence de 746 ruches-troncs $(73,4 \%)$ pour 270 ruches à cadres (recensement incluant tous les détenteurs de ruches, Bonnet 1973). La progression de la ruche moderne a ainsi été assez lente à cette époque dans le monde apicole cévenol et ce n'est que récemment que l'implantation de ruches modernes s'est accélérée : en 2014, moins d'une dizaine de ruches-troncs étaient encore peuplées par des abeilles pour tout le territoire de ces 6 communes...

\section{L'après seconde Guerre Mondiale : une période charnière}

Pendant les années d'après-guerre, de nombreux documents des archives départementales témoignent de l'intérêt de la population lozérienne pour l'apiculture moderne. Les archives départementales de Lozère ont notamment conservé les échanges entre des particuliers demandeurs d'informations et les réponses à chacun par l'ingénieur des Services agricoles de la Lozère. Ces demandes concernent la création et le développement d'élevage moderne d'abeilles, donc avec des ruches à cadres (ADL 1A à 4A) (Figure 8). Les efforts de formation, initiés durant la guerre, se poursuivent, et des spécialistes apicoles, agréés par la direction des services agricoles, se partagent le territoire en rayons d'actions et organisent la formation à l'apiculture moderne (ADL 1S à $7 \mathrm{~S})$. 
Figure 8 : À gauche, une demande de Mr. Paul Flayol à Saint Roman de Tousques pour des conseils techniques pour la mise en place d'un rucher moderne. À droite, la réponse de Mr. Quet, Ingénieur des Services Agricoles à Mende. Janvier 1947 (ADL 2A)

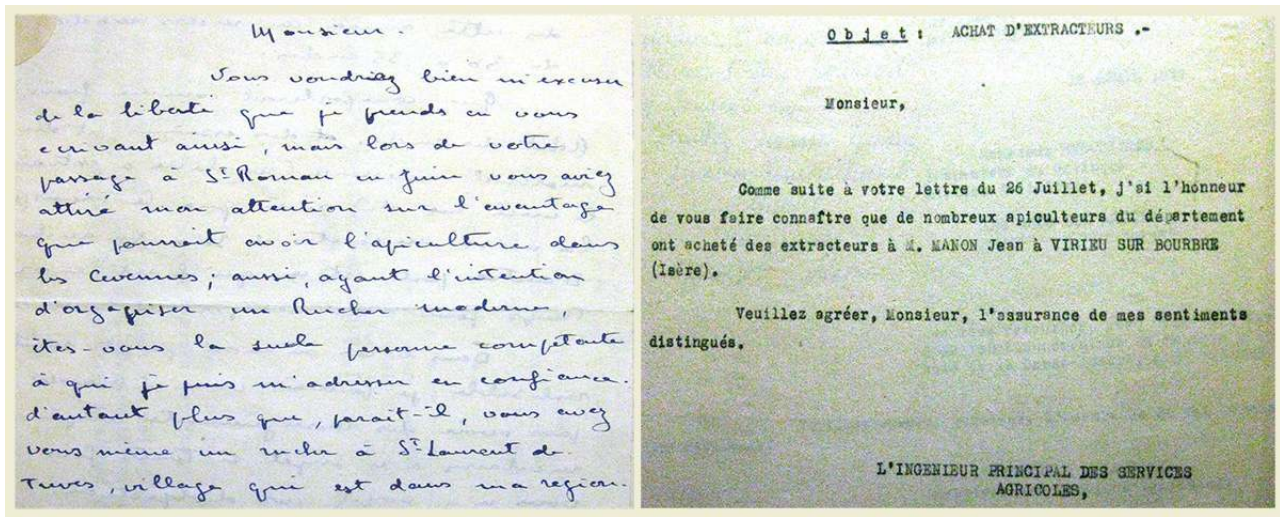

«Tout a transversé ! », expliquait une vielle dame pour expliquer les changements entre la vie avant-guerre et celle d'après. Comme tant d'autres choses, le rucher cévenol n'a pas échappé aux grands bouleversements. L'arrivée de la ruche à cadres, timide dans ses débuts a, en quelques décennies, radicalement changé le contexte apicole un peu partout dans le monde, et donc dans les Cévennes. Plutôt que d'acheter une ruche moderne déjà toute faite et qui coûtaient assez cher pour l'époque, les anciens ont d'abord pensé à ajouter une hausse sur leurs ruches-troncs (Figure 9). C'est ainsi que de nombreux apiculteurs cévenols ont tenté l'installation sur les ruches-troncs de hausses plus ou moins ingénieuses : en troncs avec des petits cadres, en paille de tout type, de ruche à cadres avec une planche percée adaptée au calibre intérieur du tronc... Peu concluantes, toutes ces tentatives furent petit à petit abandonnées ou laissées en l'état...

Figure 9 : À gauche, interview d'un ancien apiculteur en ruche-tronc réalisé par A. Lehébel-Péron, printemps 2014. À droite, une ruche-tronc avec une hausse de ruche à cadres, située en haut à droite de l'image. Rucher de la lauze (Lozère)

Jean : On avait essayé d'y mettre une hausse, de faire des cadres et mettre une hausse dessus, mais elles montaient mal dedans.

Ameline : Vous aviez fait ça quand?

J. : Quand la ruche à cadres est sortie, on en avait pas nous autres à l'époque. Et on avait essayé comme ça, mais ça marchait pas... ça montait pas bien. Je sais pas d'où ça venait. Peut-être qu'il y avait pas assez de passage, parce que dans la ruche à tronc, elles ont tendance à monter sur le bord du tronc. Et alors quand elles arrivaient, et on laissait qu' un petit trou là-haut à la cime, il fallait qu'elles se retournent comme ça, peut-être c'est ça qui les gênait. Parce que dans les ruches à cadres, il y en a qui les montent assez haut. Je pense que c'est ça qui faisait mal marcher leurs affaires.

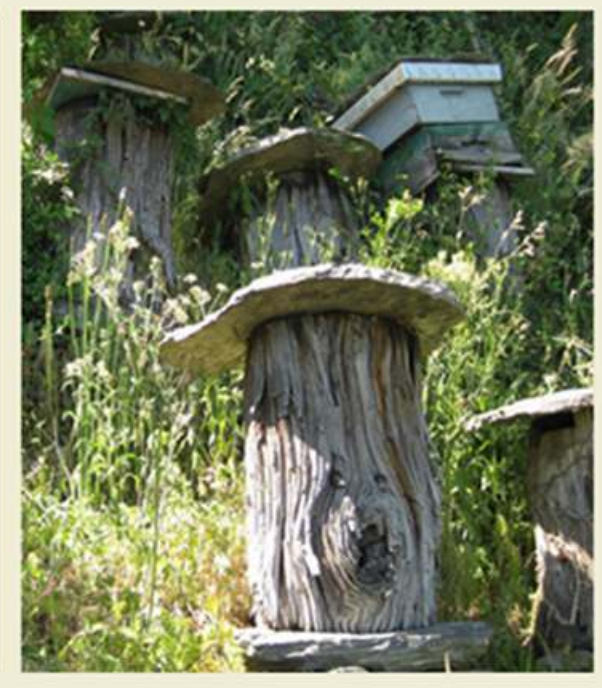

(c) A. Lehébel-Péron

Parallèlement, l'influence des revues agricoles et apicoles, l'insistance des organismes officiels, les aides techniques à l'installation et les stages de formations finissent de saper 
les dernières résistances à l'achat de la ruche à cadres. Face à la dépense, certains apiculteurs bricoleurs parviennent à fabriquer leurs propres ruches à cadres (Figure 10a)... et le rucher cévenol amorce progressivement son changement. Quelques apiculteurs détruisent leurs anciennes ruches, mais la plupart décident de les garder, même sans les exploiter, puisqu'elles sont là et qu'elles fonctionnent à moindre investissement.

Le gain de production s'en ressent d'un facteur d'environ 10, passant de 1 à $3 \mathrm{~kg}$ pour une ruche-tronc à au moins $20 \mathrm{~kg}$ (de 15 à $40 \mathrm{~kg}$ ) pour une ruche à cadres (Figure 10c). L'écart de production s'explique en partie par la mobilité de la ruche à cadres et par la capacité de stockage des hausses. Avant le démarrage de la floraison locale, ou dès la fin d'une floraison saisonnière, il est possible de déplacer les ruches au gré des sites de floraison (Figure 10b) afin d'allonger la durée du butinage et de l'intensifier par des floraisons abondantes. L'augmentation des volumes de miel, leur spécialisation florale et les revenus désormais raisonnables induits par la transhumance encouragent également une partie des apiculteurs à se "professionnaliser" et à investir en conséquence.

Figure 10 : En haut et au centre, interview d'un ancien apiculteur en ruche-tronc réalisée par Daniel Travier et Ameline Lehébel-Péron. En bas, interviews d'apiculteurs en ruche-tronc réalisées par Ameline Lehébel-Péron. Printemps 2014

Yvon : Mon père avait une tante qui avait que des ruches à troncs. Et quand il est revenu de l'armée, eh bé, l'apiculture l'intéressait. Alors il est allé voir sa tante qui avait ce rucher à troncs.

Daniel : Ton père est revenu de l'armée, c'était en quelle année ?

Y. : Alors, il est né en 5 , donc en 25 grosso modo... Donc ça lui a plu et il s'est mis à déloger les abeilles des troncs pour les incorporer dans des ruches à cadres. Et alors, hé hé, les ruches à cadres... !

Ameline : Les ruches à cadres, il y en avait beaucoup déjà ?

Y. : Bah, il y en avait point ! Et il en avait pas !

A. : Et d'où il connaissait ça?

Y. : Il y avait ici, dans la région un nommé Théophile Merry qui habitait à La Bastide, en face Peyrolles. C'était un gars resté vieux garçon et qui avait beaucoup étudié. Il avait des châtaigniers. Et lui il voulait changer, c'était un précurseur. [...] Et alors mon père, avait pris contact avec lui et il lui avait dit de toute façon si tu veux faire de l'apiculture moderne avec des ruches à cadres, il te faut faire des ruches à cadres, ou tu les commandes! Mais tu les commandes... euh, quand on a pas l'argent, c'est bien beau de dire de commander ! Et lui avait eu cette opportunité d'avoir les billes de châtaignier. Et donc les troncs de châtaigniers, les beaux, ils les avaient débités en "scieur de long ". Et avec les planches, ils avaient fait des ruches à cadres

A. : Il avait vu ça quelque part?

Y. : Dans des revues ! C'était un gars vraiment à la pointe, à la pointe de tout ! Donc il avait fait des ruches à cadres, et alors à ce moment-là, il avait pas de machine de menuiserie. Et donc rien de plus simple, quand il y avait truc qu'il voulait relier et bien, rien de plus simple, il en mettait un par-dessus et il clouait. Et donc, il avait des planches de châtaignier qui faisait deux centimètres d'épaisseur ! On aurait dit des pieds d'armoire! Et la ruche elle-même, elle faisait 60 kilos ! Ha ha ha!

D. : Et il les transhumait pas ?

Y. : Ah bah non, il les transhumait pas, i' pouvait pas! Mais quand il les a vendues, il les a transportées sur un char à boeufs pour les livrer ! Ha ha ha! 
Yvon : C'était une grande remorque, pour charrier des ruches... Vous vous rendez compte ! Et malgré ce, on en mettait une vingtaine dans cette remorque, et on pouvait pas les mettre les unes sur les autres. Et alors mon père, chez le carrossier, il avait fait couper la camionnette, et garder la cabine ! Pour faire de la transhumance !

Ameline : Là, on est dans les années...?

$\mathrm{Y}:$ Dans les années 50 et quelques, le ver à soie fonctionnait pas mal non plus. Entre la camionnette et la remorque, on faisait de ces chargements ! Mon dieu, quel temps !!

$\mathrm{D}:$ Et il transhumait où?

$\mathrm{Y}$ : En bas, à Valflaunès. Il y avait pas mal de romarin à ce moment-là. On démarrait sur le romarin en bas.

A : C'était rare les gens qui transhumaient ?

Y : Ah oui !! Mon père était un des premiers ! À part Nicolas de Saumane, eh bé, il y avait personne d'autres qui faisait de la transhumance !

Ameline : Pourquoi vous pensez que les gens ont abandonné les ruches-troncs ?

Jean : Ah, c'était la quantité ! Oui, la quantité ! Il y avait des gens, à la ruche à cadres en moyenne, il faisait une vingtaine de kilos de miel par ruche, alors...

A : Mème avec un gros tronc, on ne peut pas atteindre cela ?

$\mathrm{J}$ : Oh non, non. Au maximum, deux trois kilos, c'est tout.

\section{Aujourd'hui} sont des ruches à cadres. Quelques rares ruchers de ruches-troncs persistent dans le paysage mais sont majoritairement laissés à l'abandon. Seuls $5 \%$ des ruches hébergent une colonie d'abeilles. Il existe pourtant un regain d'intérêt à l'égard de ce type de ruche essentiellement pour leur intérêt patrimonial et potentiellement ${ }^{2}$ sanitaire (LehébelPéron 2014). Trois groupes de personnes en détiennent encore : 1) des personnes âgées (plus de 70 ans) qui possèdent et parfois entretiennent leur ancien rucher ; 2) des apiculteurs amateurs ou professionnels possédant les deux types de ruches avec des niveaux variés d'utilisation; et 3) des pluriactifs entretenant des ruches-troncs à des fins touristiques ou patrimoniales (Lehébel-Péron 2014). Quelques 190 apiculteurs répartis sur les 152 communes composant l'aire optimale d'adhésion du PnC ont déclaré leurs ruchers en 2012. Ces déclarations totalisent 732 emplacements de ruchers et 24657 ruches (Jobard 2012). Cependant, bien que la déclaration de ruchers soit rendue obligatoire depuis 2010, tous les apiculteurs ne sont pas déclarés, loin s'en faut, notamment ceux qui possèdent les plus petits ruchers. D'après les experts de la filière apicole, il existerait 3 détenteurs non déclarés pour 2 détenteurs déclarés dont le cheptel va de 1 et 10 ruches, et 2 détenteurs non déclarés pour 3 détenteurs déclarés possédant de 11 à 70 ruches. Si ces estimations sont avérées, le nombre d'apiculteurs ayant leur base d'exploitation au sein du PnC, serait compris entre 300 et 320 et le nombre total de ruches avoisinerait 26000 . Ces chiffres ne prennent pas en compte le nombre inquantifiable d'apiculteurs qui viennent transhumer dans le PnC et qui déclarent leur activité dans la commune de leur siège d'exploitation située hors du Parc.

Contrecoup de sa valeur patrimoniale et de son indéniable esthétique, la ruche tronc à un prix d'achat $(300 €)$ dorénavant trois fois plus élevé que celui d'une ruche à cadres qui est plus facile à se procurer. Le début $d u x{ }^{e}$ siècle marque l'émergence d'un attrait touristique pour les ruches-troncs, avec ses conséquences indésirables (pillage de ruchers et vente de ruche-tronc comme objet de décoration) mais aussi et surtout un fort regain 
d'intérêt patrimonial au sein du PnC. Des associations de valorisation de la ruche-tronc voient le jour, comme l'Association de Sauvegarde et de Développement du Patrimoine Apicole Cévenol (l'ADSPAC) créée en 2005 et qui a initié les actions de restauration de ruchers-troncs, et comme l'association L'arbre aux abeilles (www.ruchetronc.fr). Créée en 2008, cette dernière est désormais très active dans la conservation locale de l'abeille noire en Cévennes et dans la restauration et le repeuplement des ruches-troncs, ainsi que dans la communication sur les difficultés inhérentes à cette patrimonialisation.

\section{Historique des races d'abeilles élevées en Cévennes}

L'abeille mellifère, Apis mellifera, apparait au Paléolithique, 8 à 6 millions d'années BP, (Garnery et al. 1991, Sheppard \& Berlocher 1989, Engel 1999, Arias \& Sheppard 1996, 2005). Sa grande capacité d'adaptation a permis à l'espèce de se répandre sur une large zone géographique du Moyen Orient à l'Afrique et l'Europe. Elle vit alors à l'état sauvage dans les arbres creux ou dans les cavités rocheuses. Selon les auteurs, de 26 à 29 sous-espèces d'abeilles ont été décrites aujourd'hui sur la base de caractères morphologiques, génétiques, écologiques et comportementaux (Engel 1999, Sheppard \& Meixner, 2003, Rortais et al. 2010). En France, la sous-espèce indigène est Apis mellifera mellifera ou abeille noire. À l'époque gallo-romaine, seuls les écrits de Sidoine Apollinaire suggèrent une collecte de miel en Cévennes dès le Ve siècle (Chaix 1866, Cabanel 2009). À ce jour, l'écotype landais est l'unique écotype d'abeille qui ait été décrit et défini scientifiquement en France (Perrier et al. 2003, Strange et al. 2007). Bien que l'écotype cévenol (ou l'abeille noire cévenole) soit communément évoqué localement, il n'existe aucune preuve scientifique tangible de son existence (L. Garnery comm. pers., Lehébel-Péron 2014).

Depuis l'époque gallo-romaine jusqu'à la seconde Guerre Mondiale, c'est cette forme autochtone qui est utilisée en apiculture cévenole, de manière probablement exclusive ; à tel point que le terme d'“abeille noire" n'est quasiment jamais utilisé dans les premiers ouvrages d'apiculture moderne jusqu'au début du xxe siècle (Caillas 1946, Layens \& Bonnier 1898). Il est plutôt fait mention d'“abeille commune", consensuellement considérée comme la meilleure pour l'apiculture locale car elle est "adaptée et parfaitement acclimatée [...] prolifique, ardente, active et essaime sans exagération et élève son couvain de bonne heure et tard dans la saison [...] d'un caractère doux [...] et permet de bonnes récoltes" (Caillas 1946). En Cévennes, cette description est partagée dans le discours des personnes âgées lorsqu'elles évoquent leurs souvenirs de la première moitié $\mathrm{du} \mathrm{xx} \mathrm{x}^{\mathrm{e}}$ siècle. L'abeille qui peuple alors les ruches-troncs, est l'abeille locale, mais il n'est pas souvent question de l'abeille noire à proprement parler.

31 C'est après la seconde Guerre Mondiale que l'adoption lente et progressive des ruches à cadres va permettre aux apiculteurs d'introduire des abeilles non indigènes. Parmi cellesci, l'abeille italienne arrive en premier. Connue en France dès la fin du XIX ${ }^{\mathrm{e}}$ siècle, elle est décrite comme « l'une des plus fécondes et plus actives » (Noel 1893). Dès le début du xxe siècle, à l'initiative de la célèbre entreprise Manufrance (créée en 1885), une impulsion massive de commercialisation de l'abeille italienne est conduite sur l'ensemble de l'hexagone (Figure 11a). L'édition de 1932 du catalogue de ce fleuron français propose côte à côte les deux races d'abeilles. Commence alors une période de mixité, avec l'apparition sur le marché d'abeilles hybrides et de ruches transformées (Figure 11b).

Entre 1970 et 2007, le nombre de colonies d'abeilles sur le territoire européen périclite de plus de 21 millions à environ 15,5 millions (FAO 2009, Aizen \& Harder 2009). Les 
apiculteurs d'Europe et d'Amérique du Nord doivent faire face à des pertes hivernales répétées et causées par une combinaison de facteurs environnementaux: pesticides, impact de l'agriculture intensive, varroas et divers agents pathogènes... (Oldroyd 2007, Vanengelsdorp \& Meixner 2010). Au cours des dernières décennies, pour compenser ces effondrements de cheptel, les apiculteurs doivent recourir à l'importation des races non locales qui fait peser un fort risque d'homogénéisation des populations indigènes d'Apis mellifera mellifera (Garnery et al. 1998, Franck et al. 2000, De La Rua et al. 2009, Jensen et al. 2005). Des sous-espèces non locales comme l'abeille italienne (A. m. ligustica) ou l'abeille carnoienne (A. m. carnica), ainsi que des souches hybrides comme l'abeille Buckfast sont certes choisies en fonction de leurs caractéristiques comportementales et productives (Ruttner 1988) (Figure 11c), mais aussi en raison de leur meilleure disponibilité et de leur moindre coût (Bertrand 2013).

Figure 11 : En haut, vente par correspondance d'essaims et de reines d'abeilles noires et d'abeilles italiennes, Manufacture Française d'Armes et Cycles, Saint-Etienne Loire, 1931. Au centre, un extrait du Bulletin de la Société d'Agriculture du département de la Lozère, Tome XLVIII, 1896 (Conze 1896). En bas, un extrait de l'ouvrage de vulgarisation Le rucher durable suggérant la possibilité de sélectionner les abeilles en fonction de leur caractère (Riondet 2013).

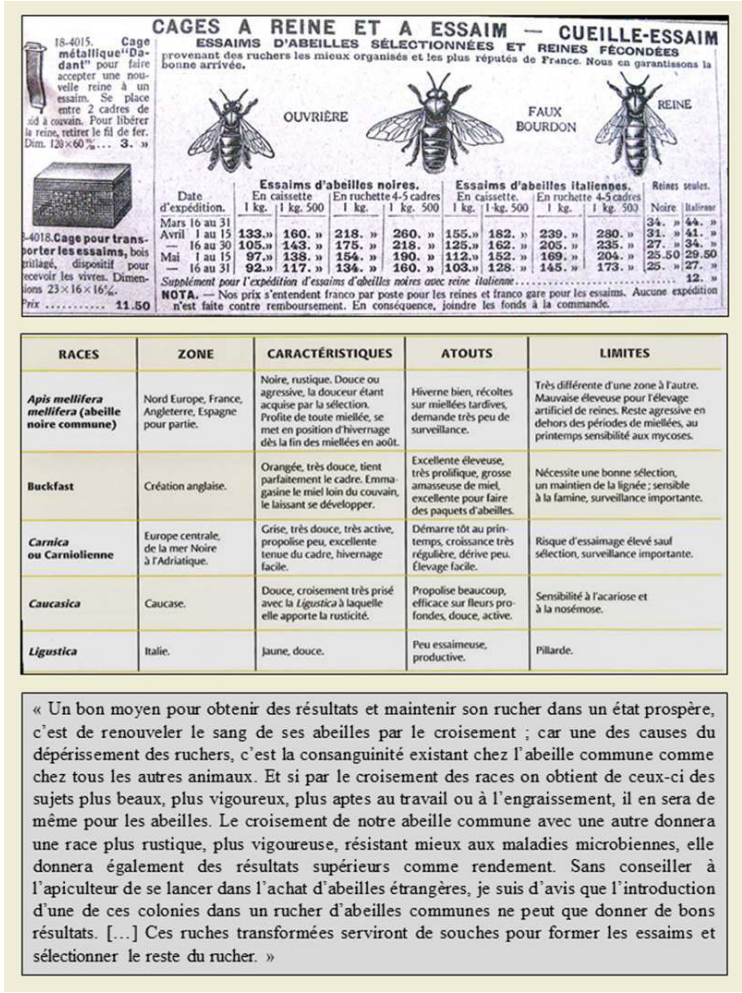

33 Jusqu'à l'imminence de la seconde Guerre Mondiale, les témoignages d'anciens apiculteurs s'accordent à dire que l'abondance des essaims spontanés suffisait à compenser les pertes hivernales éventuelles (Lehébel-Péron 2014, Clément 2015).

«Jean : On ramassait les essaims, chaque année il y avait des tas d'essaims, il y en avait qui partaient. J'ai ramassé les essaims, total que j'en avais monté une soixantaine. Et ça avait marché jusqu'à... j'avais 55, même 60 ans quand encore ça marchait. Tandis que maintenant ... ».

Les mortalités annuelles étaient des plus réduites, le plus souvent autour de $5 \%$ (Clément 2015). 
"Yvon : peut-être il y avait avant beaucoup de ruches qui étaient décimées, mais cela ne se voyait pas du fait de l'abondance des essaims qui recolonisaient les ruches vacantes. Une ruche qui se vidait, une autre qui se recolonisait, cela pouvait apparaître comme un ordre des choses ».

\section{(1)} interrogés avaient déjà remarqué une baisse du nombre d'essaims avant l'arrivée massive des importations d'abeille caucasienne en 1980 et du varroa, dans les années 1980-1982. Comme partout en France et dans le monde, le déclin massif des colonies d'abeilles se fait fortement sentir depuis 2005 en Cévennes. Dans les ruches à cadres, les apiculteurs cherchent plutôt à maîtriser l'essaimage, en réalisant des essaims artificiels (division des colonies et élevage de reines). Avec une telle raréfaction des essaims, les ruches-troncs ne parviennent pas à se repeupler, celles qui meurent ne sont plus systématiquement remplacées... les ruchers se vident.

37 Aujourd'hui, tout apiculteur a ses préférences en matière de race d'abeilles. Cependant, il renouvelle chaque année son cheptel apicole pour compenser les pertes hivernales en achetant à des producteurs d'abeilles, le plus souvent hors de sa région car trop peu de producteurs d'abeilles sont présents en Cévennes. La diversité variétale est ainsi spatialement diluée et est propice à une forte hybridation entre races d'abeilles. Par l'entremise d'une étude dédiée (Lehébel-Péron 2014), nous avons pu estimer à $30 \%$ le taux moyen d'introgression génétique (marqueur de l'hybridation) en Cévennes. Sur le terrain, chaque apiculteur dispose d'un phénotype plus ou moins précis de l'abeille noire mais le niveau d'hybridation semble être mal apprécié (Figure 12). 
Figure 12 : Deux interviews d'apiculteurs sur l'identification des abeilles noires par A. LehébelPéron, Printemps 2014.

Ameline : C'était quoi comme abeille ?

Jean : Bah euh... ça doit être... C'était pas la noire ça ... C'était l'abeille du pays.

A. : C'était pas l'abeille noire ?

J. : Non. Elle était pas noire noire... C'était l'abeille qu'on avait ici dans la région, toutes étaient à peu près les mêmes.

A. : Elle avait des caractéristiques ? Comment vous la reconnaissiez ?

J. : Oui, c'était toutes les mêmes. Ça s'est mis à changer quand il y a eu de la transhumance... Une fille de Montvert me disait cette transhumance, les bourdons avec les abeilles d'ici, ça fait une chose qui marche pas.

A. : Vous voyez la différence entre l'abeille de maintenant et celle qu'il y avait avant ?

J. : Elles sont un peu plus noires. Tandis que celles qu'on avait ici, elles étaient presque pas jaune m'enfin elles étaient claires.

A. : Et à part la couleur il y avait une autre différence ?

J. : Non... Y en avait des plus méchantes que d'autres. On savait pas d'où ça venait.

Yvon: L'abeille noire a pas disparue, mais enfin elle a beaucoup régressé, du moins il y a beaucoup de croisement. Dans ma vie de professionnel d'apiculture, j'en ai vu que deux pures!

Ameline: Noire?

Y. : Oui et c'est beau !

A. : Et comment vous les identifiez ?

Y. : L'abeille noire elle est noire et elle est plus velue qu'une abeille normale. Elle a pas des grands poils, mais on dirait du velours! Du velours noir. Mais c'est beau, hein! C'est très très beau une reine pure noire. Mais bon la majorité sont métisses, il y a eu des croisements, je veux dire des quantités !

\section{Historique des produits de la ruche}

Nous n'avons pas recueilli d'informations concernant la nature des produits de la ruche avant le Moyen Âge, même s'il est fortement probable qu'il s'agisse au moins de miel (Roffet-Salque et al. 2015).

\section{Le Moyen Âge}

L'apiculture cévenole a connu un tel développement du Moyen Âge jusqu'au xive siècle que ses produits ont pu être utilisés comme monnaie, notamment pour l'acquittement du cens qui était une redevance annuelle fixe que le possesseur d'une terre devait au seigneur. Cet usage démontre à la fois l'importance et la régularité de la production. Ainsi, les premiers témoignages que l'on doit au recueil des actes en Vallée Française par Terrier de la Boissonnade (Archives du Lien des Chercheurs Cévenols, www.cevenols.fr) comprennent notamment un acte de 1353 où le cens est d'« une livre de cire », et un acte de 1371, où le cens est d' " un carteron de miel mesure de Moissac ». Le carteron médiéval est une mesure de capacité similaire à la pinte de Châlus qui équivalait au $\mathrm{XVIII}^{\mathrm{e}}$ siècle à 2,380 litres à Barre-des-Cévennes. Dans un acte de 1504, il est question de «cens d'une demi-livre de cire ». Il s'agit ici de «livres grosses médiévales » (411,13 g à Montpellier) qui deviennent «livres poids de table » aux Temps Modernes, c'est-à-dire 414,65 g à Montpellier (D. Travier comm. pers.). Le cens est payé aussi bien en miel qu'en cire car ces deux produits de la ruche ont localement une valeur économique importante dans la seigneurie. 


\section{Du XVe au XVIII ${ }^{\mathrm{e}}$ siècle}

40 main, ou par torsion dans un torchon à maille relativement lâche et par pressage manuel. Une quantité importante de cire est extraite des parois des rayons de miel confectionnés par les abeilles. Le commerce de la cire recourt à une terminologie particulière ; par exemple le mot esquichos correspondant aux pelotons de cire brute et issus du verbe occitan eskichar signifiant "presser" (Boissier de Sauvages 1785). La cire d'abeille a une valeur équivalente à celle du miel, tant dans le «Capitulaire de Villis » édicté par Charlemagne que dans les actes seigneuriaux cévenols où elle est intégrée dans le cens. La cire d'abeille, comme le suif de bœuf ou de mouton, est essentiellement utilisée dans la fabrication de chandelles qui, depuis le Moyen Âge, rivalisent avec les lampes à huile. Ces dernières réclament une attention constante tandis que les chandelles, constituées d'une mèche entourée d'une matière combustible solide et fondante, éclairent mieux, brûlent plus lentement et ne sentent pas mauvais (D. Travier comm. pers., Crane 1999, LehébelPéron 2014). En 1426, Henri VI, Roi d'Angleterre, confirme dans ses ordonnances en tant que Roi de France les statuts de la corporation des chandeliers de Pontoise; en pleine guerre de 100 ans, cet acte de loi souligne le poids économique de l'activité des faiseurs de chandelles. Il existe des cires d'abeille de différentes qualités : la cire brute ou jaune provient de la fonte des parois des rayons par trempage dans l'eau chaude et élimination des corps étrangers; la cire blanche est obtenue en décolorant la cire jaune par une exposition au soleil. Grâce à des règlements de fabrication très stricts (interdiction de mélange avec d'autres matières comme le suif, la graisse, la poix ou la résine) édictés par les corporations des maîtres chandeliers, les cierges en cire d'abeille acquièrent à l'usage une meilleure réputation que les chandelles de suif. $\mathrm{Au} \mathrm{XV}^{\mathrm{e}}$ siècle, la surveillance scrupuleuse de la fabrication et de la vente des chandelles en cire d'abeille induit un surcoût que seuls la noblesse et le clergé sont en mesure de supporter. Plusieurs documents de cette époque montrent l'importance économique du miel et de la cire et son développement en Cévennes comme dans le reste du territoire français (Figure 13). Vers 1760 , le prix d'une chandelle de cire équivaut à une demi-journée de travail d'un manœuvre (Figure 13a). Une vente du 10 février 1765 à Saint Etienne Vallée Française en Cévennes apporte des éléments sur leur utilisation avérée en pharmacie (Figure 13b). Le notable Pierre de Gervais possède des abeilles et se les réserve. Il vend le miel à l'apothicaire qui en a l'exclusivité et qui l'utilise très certainement à des fins médicinales. Sieur de Maroul est un gentilhomme propriétaire foncier qui a des terres et des fermiers. Les levées de miel et de cire dans ses borgnons ont lieu deux fois chaque année, au printemps et à la fin de l'été, au mois de mars et au mois d'août ou septembre. Un quintal (environ 40-41 kg) se vend 15 livres, ce qui atteste de la rentabilité de cette exploitation. Est également présenté un acte par lequel un vassal reçoit une terre en fief (Figure 3c). Il verse à son seigneur une somme d'argent et un impôt en nature correspondant à un ancien droit de gîte, l'albergue très symbolique correspondant à une once $(26 \mathrm{~g})$ de cire blanche. Les ruches interviennent en outre comme éléments de dot de mariage ou de legs testamentaires (Figures 13d \& 13e). 
Figure 13 : Documents spécifiant la place de l'activité apicole dans la vie courante. a. Coût de la vie, prix du miel et de la cire (Guillou 1918, Chaze 2012). b. Acte de vente de miel et de cire (1765). c. Inféodation pour 650 livres et « l'albergue " d'une once de cire blanche (1751). d. Contrat de mariage avec en dot en essaims d'abeilles avec leurs ruches (1764). e. Testament spécifiant un legs de ruches à miel (1676)

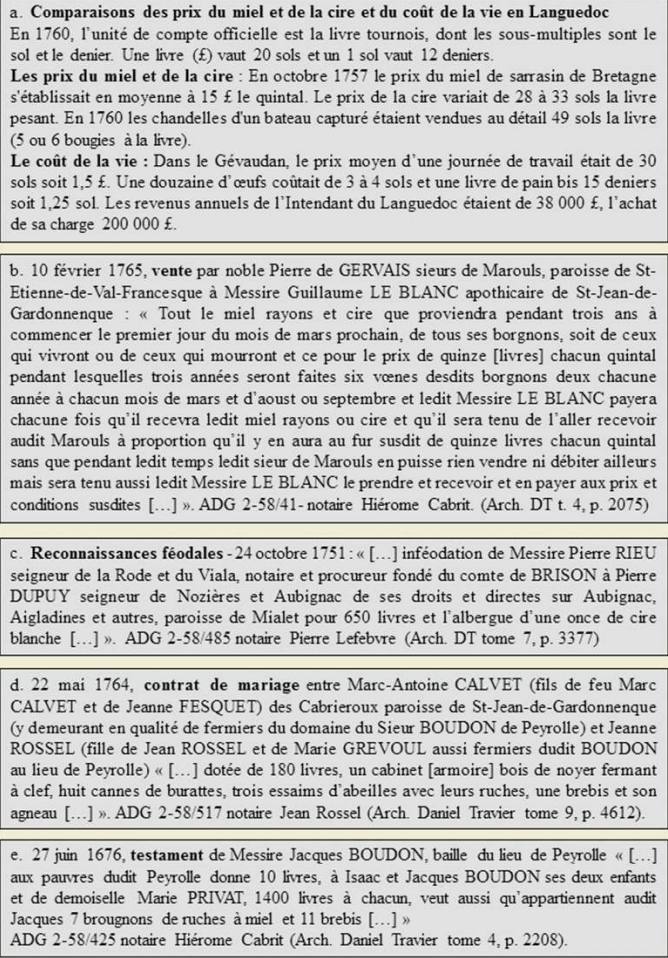

41 Il existe ainsi dans les langues populaires, notamment cévenoles, un vocabulaire spécifique à l'abeille et aux ruches, de nombreux toponymes avec un rapport à l'abeille, une présence de l'abeille dans les contrats de mariage et les testaments. Il faut toutefois attendre le début du $\mathrm{XVII}^{\mathrm{e}}$ siècle pour assister à la diffusion des premiers ouvrages scientifiques sur ce thème. Le premier d'entre eux intitulé le " Théâtre de l'agriculture et ménage des champs" d'Olivier de Serres parait en 1600 et fournit beaucoup de renseignements sur la pratique apicole en Cévennes (assimilant partiellement l'Ardèche) de la fin $\mathrm{du} \mathrm{XvI}^{\mathrm{e}}$ siècle. Très répandu dans les Cévennes protestantes, cet ouvrage indique par exemple que pour les ruches, « on préfère le bois à la paille, la terre cuite ou la pierre et qu'on préfère les ruches monoxyles à celles menuisées » (D. Travier comm. pers.). Sont ensuite publiés en 1701 "L'économie générale de la campagne ou nouvelle maison rustique » de Louis Ligier de la Bretonnerie et constamment réédité au XVIII ${ }^{\mathrm{e}}$ siècle (Liger \& Bernier 1775) puis en 1837 «Le cours complet d'agriculture» de l'abbé Rozier, encore présents dans les bibliothèques de quelques propriétaires fonciers cévenols (D. Travier comm. pers.).

\section{Du XIX siècle à l'avant seconde Guerre Mondiale}

Le début du siècle est encore marqué par le négoce de la cire, comme le montre une correspondance datée de 1834 entre des courtiers dont l'un est établi à Toulouse et l'autre dans le Gard à Saint André de Valborgne (Figure 14). Ce dernier n'est qu'un intermédiaire, qui ne produit rien mais achète puis revend la cire en mettant en relation des fabricants 
de cierges et de bougies toulousains avec un producteur établi à Florac. D'autres passages de cette correspondance non présentés ici montrent que même si la cire est l'objet d'un véritable négoce, sa production et sa récolte au printemps et en fin d'été restent encore très artisanales et aléatoires.

Figure 14 : Lettres de négoce de cire (1834) Archives D. Travier crise de la sériciculture ainsi que par la révolution industrielle qui condamne l'agriculture traditionnelle ; c'est aussi le moment d'arrivée de la maladie dite de l'encre qui affecte les châtaigniers. Ainsi, de 1841 à 1911, le village de Saint-Germain-de-Calberte perd près de la moitié de sa population. Les Cévenols s'orientent vers le secteur tertiaire des grandes entreprises, administrations et banques (Ponts et chaussées, chemins de fer, Poste, Éducation Nationale, banques...) en milieu urbain. Par contre, ce sont les paysans des régions voisines comme ceux des hautes terres du Gévaudan et Margeride, de la Haute Loire et du plateau ardéchois qui s'orientent vers le bassin houiller d'Alès. Au cours de cette même période, des villes de ce secteur comme la Grand Combe gonflent de 4000 à 11 500 habitants et Alès de 16000 à 30000 habitants (recensement national de la population, Musée des vallées cévenoles). Dans pareil contexte, il est hautement probable qu'aucun nouveau rucher n'ait été implanté et construit après 1850, mais il est certain que la quasitotalité du miel est issu des ruches-troncs (D. Travier comm. pers.).

C'est dans ce contexte de transformations profondes de la société en fin de xix ${ }^{e}$ siècle, que sucre et café font leur apparition en Cévennes, mais ne sont alors consommés que par les notables et les personnes de rang social élevé. Cette consommation reste cependant anecdotique. Privé de ces nouveaux produits, le peuple se doit de maintenir une production abondante de miel, avec lequel il sucre tisanes et infusions qu'il consomme abondamment.

«Dans ma famille, on buvait le café seulement deux fois par an, quand on décoconnait, parce que c'était une fête où on invitait les voisins et on allait avoir 
quatre sous des cocons et puis peut-être pour la Noël ou pour le jour de l'an. Le sucre c'était pareil. On avait des pains de sucre, mais au quotidien le sucre qu'on consommait c'était le miel »(D. Travier témoignage).

Les recensements agricoles des archives départementales de la Lozère rendent compte de l'évolution du nombre de ruches, de la production et du prix de la cire et du miel sur près d'un siècle et avant la seconde Guerre Mondiale (ADL 1ST à 3ST). La figure 15 illustre tout d'abord la difficulté de documenter cette activité apicole au vu du nombre d'années sans information alors que ces enquêtes sont réalisées deux fois par an dans chaque commune. La baisse prononcée de toutes les valeurs (exception faite du prix de la cire et du miel à Saint-Julien-d'Arpaon) est consécutive de l'exode rural massif à cette période. Toutes les valeurs s'effondrent drastiquement, notamment d'un facteur 4 pour le nombre de ruches. Les prix varient nettement moins que les productions de cire (variation d'un facteur de plus de 60) et de miel (facteur 20), ce qui suggère une volonté de stabiliser ce marché malgré ces variations interannuelles sûrement imputables pro parte au climat. D'ailleurs, à Cassagnas, les productions de cire et miel ne sont pas significativement corrélées au nombre de ruches (cire : $\mathrm{R}^{2}=0.427, \mathrm{P}>0.05,17 \mathrm{ddl}$; miel : $\mathrm{R}^{2}=0.215, \mathrm{P}>0.10,18 \mathrm{ddl}$ où $\mathrm{R}$ : indice de corrélation, $\mathrm{P}$ : probabilité statistique, $\mathrm{ddl}$ : degré de liberté de cette corrélation). D’après les recensements de ce village, la production moyenne ( \pm SD avec $\mathrm{SD}$ : déviation standard associée à la moyenne) par ruche est de $0,72 \pm 0,24 \mathrm{~kg}$ pour la cire (min. : 0,2; $\max .: 1,25$ ) et $1,76 \pm 0,91 \mathrm{~kg}$ pour le miel (min. : 0,5; max. 3,75). Cependant, il s'agit là d'informations ponctuelles concernant uniquement deux villages, qui ne permettent pas de généraliser faute d'études plus extensives.

Figure 15 : Variations de la production et du prix (en francs) de cire et de miel entre 1862 et 1936 dans deux villages cévenols, Cassagnas (en rouge) et Saint-Julien d'Arpaon (en bleu). Le nombre de ruches est également indiqué uniquement pour Cassagnas

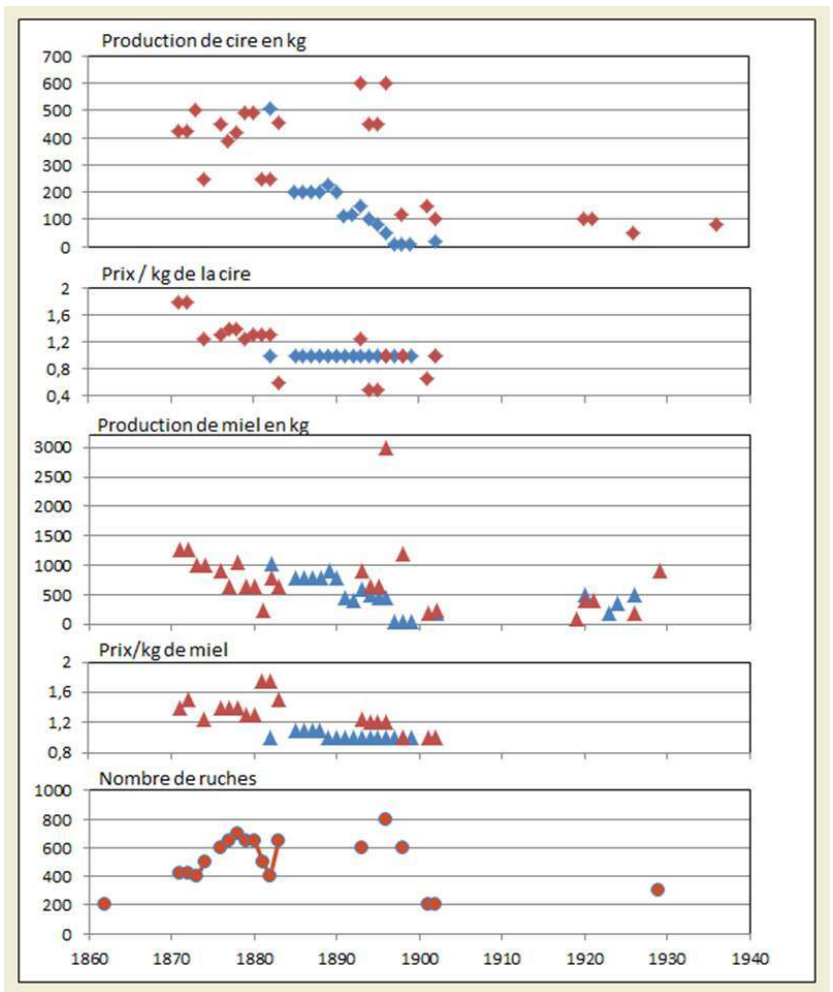

Figures établies d'après les recensements agricoles des archives départementales de la Lozère. 


\section{La seconde Guerre Mondiale et la Libération, une période charnière}

46 confrontés à des difficultés de ravitaillement et déterminent par la planification et le contrôle une transformation des pratiques qui orientera à terme l'activité apicole vers la modernité. Pendant les années de guerre, l'État français considère la production du miel comme un réel appoint au ravitaillement général de la nation. Entre 1941 et 1944, des mesures sont édictées pour accroître l'activité d'établissements apicoles existants avantguerre (ADL 8S), pour inciter au développement de l'apiculture, par son introduction dans la formation des instituteurs (ADL 9S), et pour organiser la production en planifiant la quantité de miel que chaque apiculteur doit livrer (ADL 2N). Durant toute la seconde Guerre Mondiale, le prix du miel augmente fortement (jusqu'à 5 francs/kg), ce qui occasionne des vols de miel et contraints certains apiculteurs à cadenasser leurs ruches (Clément 2015). À la Libération, les nouvelles autorités de la République ne remettent pas en cause fondamentalement ces initiatives. Outre l'interdiction de détruire des colonies par étouffage (ADL 1I), de nombreux documents des archives départementales de la Lozère portent sur la lutte contre les maladies contagieuses, notamment la loque (ADL 2D).

Durant les années de guerre, les pratiques apicoles changent par crainte de pertes de cheptel d'abeilles; le nourrissement en sucre des abeilles semble ainsi devenir courant et nécessaire. Le ravitaillement des apiculteurs en sucre impropre à l'alimentation humaine fait l'objet de nombreux courriers entre les Services agricoles et les apiculteurs (ADL 1N à $8 \mathrm{~N}$ ) (Figure 16). Toutefois, ces sources ne permettent pas de déterminer si le sucre est plus utilisé en ruches à cadres qu'en ruches-troncs. Le nourrissement était en général moins pratiqué en ruches-troncs, car le miel n'en était jamais intégralement extrait. Tout au plus les Cévenols utilisaient le bouillon de la soupe de châtaigne pour répondre aux besoins de la colonie. Certains changements de pratiques ont pu avoir des effets pervers. Ainsi, en 1941, l'incitation à utiliser du sucre de raisin désacidifié en guise de nourrissement se solde par une mortalité sévère des abeilles, obligeant l'administration à revenir sur cette disposition (ADL 9N).

Figure 16 : Lettre du Directeur des Services Agricoles à Monsieur Pigon Jean, Pasteur à SaintEtienne-Vallée-Française et à Monsieur Saint-Pierre, épicier à Montbrun - 2 novembre 1942.

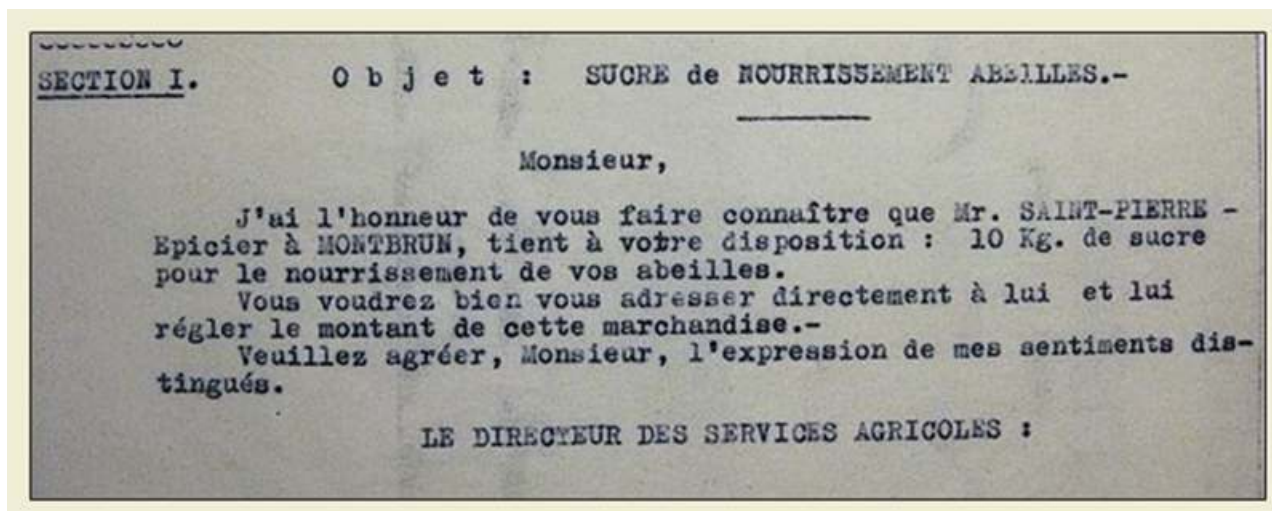

Source : ADL $1 N$

Revue d'ethnoécologie, 9 | 2016 
'avènement de l'électricité met fin aux lampes à huile et rend anecdotique l'utilisation de la cire pour la fabrication des chandelles. La plupart des bougies courantes sont à présent en paraffine. Avec les ruches à cadres, le miel est extrait sans destruction des rayons qui peuvent alors être replacés dans la ruche. Ayant beaucoup moins de cire à produire, les abeilles sont davantage productives en miel. L'implantation des ruches à cadre démocratise l'exploitation à des fins alimentaires et médicinales d'autres produits issus de la ruche comme la gelée royale, le pollen et la propolis (Bertrand 2016). En Cévennes comme d'ailleurs dans le reste de l'Europe, le miel reste néanmoins le principal produit motivant la pratique de l'apiculture. Les ruches à cadres permettent d'allonger la période de butinage mais aussi d'augmenter le nombre de miellées monoflorales. La production cévenole de miel connaît d'ailleurs actuellement un nouveau tournant de son histoire puisque la Commission Européenne a récemment avalisé l'Indication Géographique Protégée (IGP) «Miel des Cévennes» (Bulletin officiel du Ministère de l'agriculture, de l'agroalimentaire et de la forêt 2015). Justifiée par la diversité de la flore cévenole butinée par les abeilles, l'appellation dont bénéficie ce miel repose sur une production dans une zone géographique définie, mais peut être polyfloral ou monofloral (châtaignier, bruyère, callune, framboisier et ronce) (Bulletin officiel du Ministère de l'agriculture, de l'agroalimentaire et de la forêt 2015). Toutefois, la production de miel de callune, principalement sur le mont Lozère, connait des difficultés particulières liées à une combinaison de facteurs climatiques, environnementaux et économiques (LehébelPéron et al. 2016). Il convient de noter que cette IGP porte indistinctement sur les miels produits en ruche à cadres et en ruche-tronc (Bulletin officiel du Ministère de l'agriculture, de l'agroalimentaire et de la forêt 2015), ce qui est en soi une reconnaissance de la diversité contemporaine de la production et de l'histoire apicole cévenole.

\section{Discussion}

Un trait remarquable de l'apiculture contemporaine est qu'elle mobilise un large spectre de pratiques, depuis l'apicollecte de subsistance, encore en vogue sous diverses latitudes intertropicales (Dounias \& Michon 2011), jusqu'à des formes très industrialisées aux États-Unis ou en Nouvelle-Zélande. De cette coexistence de pratiques découle un vaste gradient de domestication qui s'opère sous nos yeux (Marchenay 1979, Crane 1999). Cette diversité synchronique à l'échelle mondiale se retrouve au moins partiellement dans le déroulé historique des pratiques apicoles en Cévennes (Figure 17): il débute par une apicollecte de subsistance dans les châtaigniers en prémices à une apiculture très vite renommée et inscrite dans le paysage cévenol dès le $v^{e}$ siècle. Les archives que nous avons restituées au fil de leur contexte historique rendent compte de ruchers conçus à partir de matériaux locaux, avec la confection de ruches en paille attestées depuis le XIII ${ }^{\mathrm{e}}$ siècle. S'ensuit une probable apiculture en ruches-troncs dont le premier témoignage avéré ne date toutefois que de 1627. Apparues et soutenues depuis le début du $\mathrm{Xx}^{\mathrm{e}}$ siècle, les ruches à cadres progressent plus lentement en Cévennes que dans les autres régions françaises et ne rivalisent en nombre avec les ruches-troncs qu'à partir des années 1970, avant de les supplanter définitivement dans les années 1980. Cette prédominance tardive de la ruche à cadres souligne la rémanence d'une apiculture traditionnelle en ruches-troncs dans le paysage cévenol et dans la mémoire d'une génération encore vivante de praticiens. Cette persistance bioculturelle d'une pratique traditionnelle permet encore d'en documenter la 
richesse et d'en prendre toute la mesure patrimoniale et conservatoire (Lehébel-Péron 2014). Localement dominante pendant au moins trois siècles et demi, l'apiculture en ruche-tronc a fait la démonstration de ses atouts et force le respect par sa capacité à contenir les assauts de l'apiculture dite moderne, pourtant amplement soutenue dès l'après-guerre par des campagnes publicitaires de vaste ampleur, par les structures d'encadrement agricoles et par des incitations gouvernementales.

Les différents documents et témoignages rassemblés ont permis de détailler la transformation de l'apiculture en Cévennes, qui a concerné à la fois la ruche, les abeilles, les produits de la ruche et, incidemment, le paysage cévenol (Figure 17). Les premières abeilles italiennes sont introduites en Cévennes entre les deux guerres; après la seconde Guerre Mondiale se succèdent l'introduction d'autres races d'abeilles allochtones et le parasite varroa. Cette phase de transition a été marquée d'une part par des tentatives volontaires d'"hybridation" technique entre les deux types de ruches et, d'autre part, par une augmentation progressive d'hybridations génétiques aussi involontaires qu'incontrôlées entre les différentes races d'abeilles (Lehébel-Péron 2014, L. Garnery comm. pers.). Cette transition s'est accompagnée d'une profonde transformation des pratiques apicoles locales (les premiers nourrissements au sucre voient le jour durant la seconde Guerre Mondiale), avec parfois même des tâtonnements dans les recommandations administratives. Ces transformations des pratiques voient l'émergence des premiers apiculteurs à part entière, capables de vivre de la seule activité apicole, alors qu'avant 1970 cette pratique s'inscrivait dans la pluriactivité de la majorité des foyers. Les produits de la ruche ont également changé puisque la production de cire connut une rentabilité équivalente à celle du miel avant de sombrer dans l'obsolescence. Aujourd'hui, gelée royale, pollen et propolis offrent des débouchés alternatifs que la ruche à cadres permet de produire à un niveau nettement plus important (Bertrand 2016). 
Figure 17 : Schéma récapitulatif de l'évolution de l'apiculture en Cévennes, avec des informations concernant les ruches (en brun), celles pour les abeilles et les pratiques apicoles (en vert), celles pour les produits de la ruche (en bleu), et celles du contexte humain (en noir).

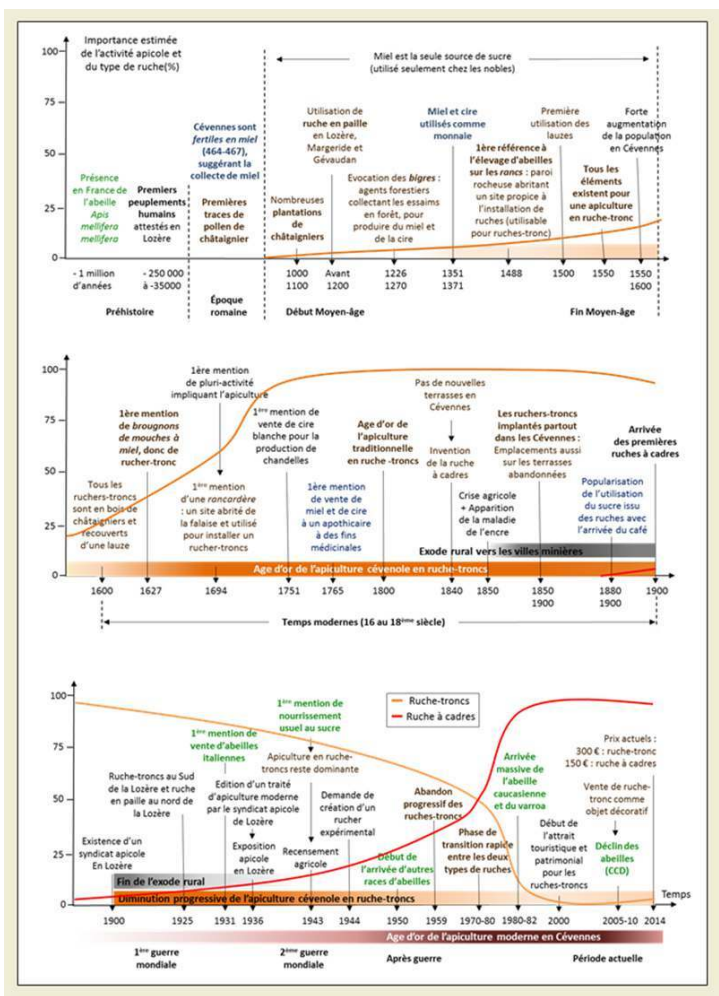

51 À l'âge d'or des ruches-troncs, l'apiculture était fixiste (l'extraction du miel impose une destruction des rayons) et sédentaire et tirait pleinement partie des ressources locales tant pour les abeilles que pour les matériaux (troncs de châtaignier et lauzes pour les ruches, terrasses en terre et pierres pour le rucher). Cette apiculture reposait sur un savoir-faire en adéquation avec les contraintes écologiques et la rudesse du milieu cévenol; ce savoir s'exprimait jusque dans la conduite de la végétation attenante au rucher, notamment par la plantation d'espèces à floraison précoce (buis, arbres fruitiers) et tardive (lierre) de sorte à allonger la saison de collecte de nectar et de pollen (LehébelPéron et al. 2015). Les pathogènes, aujourd'hui plus difficiles à contrôler en ruche-tronc qu'en ruche à cadres, étaient auparavant moins virulents, et l'abondance des essaims permettait d'en pallier les effets. Nous n'avons trouvé aucune trace d'effondrement de colonies durant la période d'âge d'or des ruches-troncs. La production en miel était relativement modeste mais couvrait les besoins alimentaires en sucre de la population cévenole, tout en dispensant un numéraire par la vente de cire. Cette apiculture avait trouvé sa place multi-usages dans la vie courante et l'économie domestique des familles cévenoles au côté des activités maraîchères, horticoles, pastorales et bûcheronnes. C'était une apiculture domestique et paysagère, optimisée dans un contexte de pluriactivité de subsistance (Lehébel-Péron 2014). Des formes parentes de cette apiculture convergeant autour de la satisfaction de besoins domestiques par la pluriactivité, persistent encore aujourd'hui dans diverses régions du monde, et particulièrement dans la zone intertropicale (Crane 1999, Roué et al. 2015).

L'apiculture moderne qui prévaut aujourd'hui en Cévennes est en tous points opposée à l'apiculture en ruche-tronc. Elle est mobiliste (les rayons sont sur des cadres mobiles), 
transhumante (permise par le déplacement des ruches et motivée par l'augmentation de production de miel) et n'utilise pas de matériaux locaux (bois manufacturé, zinc ; rucher constitué de ruches posées à même le sol ou parfois sur un support en pneus, palettes ou parpaings). Le savoir-faire s'est déporté sur la pratique apicole proprement-dite et l'apprentissage de nouveautés : choix de la race d'abeille, ouverture répétée de la ruche, observation des cadres, transhumance, suivi de la reine et croisements contrôlés... L'accroissement en nombre et en virulence des pathogènes requiert des traitements sanitaires lourds et issus dans la plupart des cas de l'industrie; les ruches doivent être repeuplées régulièrement par de nouvelles colonies achetées très généralement hors des Cévennes. L'apport indéniable des ruches à cadres est la production élevée en miel permettant à l'apiculteur de vivre uniquement de cette activité. Il s'agit d'une apiculture maximisée, soumise aux affres de la compétitivité économique à l'échelle internationale et de la loi du marché, au sein d'une filière en constante délocalisation et tenue par un cahier des charges de plus en plus astreignant.

L'ethnoécologie historique de l'apiculture en Cévennes permet une compréhension fine de la situation locale de l'apiculture et des conditions historiques de sa transformation. Elle est un élément-clé dans l'établissement des stratégies de conservation et de restauration (Swetnam et al. 1999, Egan \& Howell 2005) et elle fournit des arguments de sauvegarde des savoirs locaux en voie d'extinction. L'apiculture moderne qui s'est tardivement imposée en terre cévenole est de plus en plus mise à mal par des difficultés sanitaires en contexte de changement planétaire, dont le syndrome d'effondrement des colonies (Colony Collapse Disorder : CCD) est l'une des expressions les plus dramatiques (Cox-Foster et al. 2007). Les apiculteurs sont aujourd'hui contraints de repeupler de plus en plus fréquemment leurs ruches afin de compenser les pertes hivernales (Breeze et al. 2014). $84 \%$ des cultures agricoles en Europe dépendraient des pollinisateurs et plus particulièrement des abeilles (Williams 1994) et les évaluations du coût économique lié à la perte de pollinisation sont de plus en plus alarmantes (Gallai et al. 2009, IPBES 2016). Les problèmes sont connus, tout autant que leurs solutions qui requièrent un changement radical de modèle économique car toutes s'accordent sur la nécessité de privilégier une apiculture plus durable, ce qui concerne aussi plus généralement l'agriculture. Dans ce contexte actuel difficile, l'apiculture optimisée qui a su perdurer localement plus de trois siècles durant peut légitimement inspirer cette transition requise et $\mathrm{y}$ prendre part activement. Une ruche ronde et en bois de châtaignier riche en tanins (deux facteurs qui minimiseraient l'impact des pathogènes, Fernández-Salas et al. 2011, Bertrand 2016), et à la paroi épaisse qui atténuerait fortement les variations hygrothermiques à l'intérieur de la ruche (Lehébel-Péron 2014), inscrite dans un paysage dont la diversité floristique et faunique doit être préservée, et astreinte à une pondération des interventions par l'apiculteur et à un prélèvement modéré de miel (deux facteurs qui amélioreraient la survie des colonies, Bertrand 2016), sont autant d'atouts à faire valoir. La production d'une abeille noire en Cévennes adaptée aux contraintes environnementales locales serait également une piste à privilégier par les gestionnaires du PnC, en complément des actions de diffusion d'informations et de restauration des ruchers déjà initiées (Lehébel-Péron 2014).

Loin d'être uniquement contemplatrice, l'approche en ethnoécologie historique mobilisée dans la présente étude est porteuse d'applications concrètes associées aux valeurs- de l'apiculture traditionnelle, lesquelles pourraient être mises à contribution dans la nécessaire recomposition de la pratique apicole. Cette recomposition permettrait de 
concilier la rentabilité économique d'une filière locale aujourd'hui en régression et les enjeux de patrimonialisation défendus par le Parc national. Pareille conciliation ne peut s'envisager sans une concertation entre tous les acteurs concernés.

Cette étude a été financée par une convention CIFRE ( $N^{\circ}$ 2009/0758) entre l'Association Nationale pour la Recherche et les Technologies (ANRT, www.anrt.asso.fr) et le Parc national des Cévennes ( www.cevennesparcnational.fr). Nous remercions chaleureusement tous les apiculteurs et les personnes interrogées lors de cette étude et tout particulièrement celles et ceux qui ont vécu la fin de l'âge d'or des ruches-troncs, pour leur patiente collaboration et pour avoir partagé leurs souvenirs. Nous remercions aussi les agents $d u$ PnC nous ayant soutenus dans ce projet, avec une mention particulière à Emeric Sulmont.

\section{BIBLIOGRAPHIE}

Aizen M.A. \& Harder L.D. 2009 - The global stock of domesticated honey bees is growing slower than agricultural demand for pollination. Current Biology 19 : 915-918.

Arias M.C. \& Sheppard W.S. 1996 - Molecular Phylogenetics of Honey Bee Subspecies (Apis mellifera L.) inferred from Mitochondrial DNA Sequence. Mol. Phylogenet. Evol. 5 : 557-566.

Arias M.C. \& Sheppard W.S. 2005 - Phylogenetic relationships of honey bees (Hymenoptera: Apinae: Apini) inferred from nuclear and mitochondrial DNA sequence data. Mol. Phylogenet. Evol. 37 : 25-5. [doi:10.1016/j.ympev.2005.02.017].

Barrau J. 1983 - Les hommes et leurs aliments: esquisse d'une histoire écologique et ethnologique de l'alimentation humaine. Temps actuels.

Bertrand B. 2013 - Analyse de la diversité génétique de populations d'abeilles de la lignée OuestMéditerranéenne (Apis mellifera mellifera) : Application à la conservation. Thèse de doctorat, Université Paris Sud, Paris.

Bertrand B. 2016 - Les ruches de biodiversité. Éditions du Terran.

Blanchemanche P. 1986 - Les terrasses de culture des régions méditerranéennes. Terrassements, épierrement et dérivation des eaux en agriculture. XVII ${ }^{e}$-XIX ${ }^{e}$ siècles. Étude ethnohistorique. Mémoire de doctorat de 3e cycle, École des Hautes Études en Sciences Sociales, Paris.

Boissier de Sauvages 1785 - Dictionnaire Languedocien-Français.

Bonnet R. 1973 - Inventaire des ruches du Versant sud du Bouges (Haute vallée de la Mimente). Archives du Parc National des Cévennes.

Breeze T.D., Vaissière B.E., Bommarco R., Petanidou T., Seraphides N., Kozák L., Scheper K., Biesmeijer J.C., Kleijn D., Gyldenkærne S., Moretti M., Holzschuh A., Steffan-Dewenter I., Stout J.C., Pärtel M., Zobel M. \& Potts S.G. 2014 - Agricultural policies exacerbate honeybee pollination service supply-demand mismatches across Europe. PLoS ONE 9 (1) : e82996. doi:10.1371/ journal.pone.0082996

Brinkmann W. 1938 - Bienenstock und Bienenstand in romanishen Ländern, Hanscher Gildenverlag. ed. Hamburg. 
Brousse A. 1930 - Petit traité d'apiculture moderne. Mende, Impr. H. Chaptal.

Bulletin officiel du Ministère de l'agriculture, de l'agroalimentaire et de la forêt 2015 - Cahier des charges de l'indication géographique protégée (IGP) « Miel des Cévennes », 1 (6) : 201-205.

Cabanel P. 2009 - Histoire des Cévennes. Paris, Presses Universitaires de France.

Caillas A. 1946 - Le Rucher de rapport - traité pratique d'apiculture moderne 1ère édition.

Chaix L.A. 1866 - Saint Sidoine Appolinaire et son siècle. Thibaud.

Chevallier R. 1982 - Pline l'Ancien et la Narbonnaise. Rev. Belge Philos. Hist. 60 : 136-142.

Clément H. 2015 - L'apiculture cévenole. Revue Causses et Cévennes 120 (1) : 191-195.

Conze C. 1896 - Mémoire sur l'apiculture pratique. Bulletin de la société d'agriculture, Industrie, Sciences et Art du Département de La Lozère. Mende.

Cox-Foster D.L., Conlan S., Holmes E.C., Palacios G., Evans J.D., Moran N.A., Quan P.L, Briese T., Hornig M., Geiser D.M., Martinson V., vanEngelsdorp D., Kalkstein A.L., Drysdale A., Hui J., Zhai J., Cui L., Hutchison S.K., Simons J.F., Egholm M., Pettis J.S., Lipkin W.I. 2007 - A metagenomic survey of microbes in honey bee colony collapse disorder. Science $318: 283-287$.

Crane E. 1999 - The world history of beekeeping and honey hunting. Taylor \& Francis, USA.

Crittenden A.N. 2011 - The importance of honey consumption in human evolution. Food and Foodways 19 : 257-273.

De La Rúa P., Jaffé K., Dall’olio R., Muñoz I., Serrano J. \& De La Rúa A. 2009 - Biodiversity, conservation and current threats to European honeybees. Apidologie $40: 263-284$.

Dounias E. \& Michon G. 2011 - Le miel en forêt : apicollectes, apicultures. In : Michon G. \& Moizo B. (Ed.) Des forêts et des hommes, Suds en ligne. Les dossiers thématiques de l'IRD. [En ligne : www.suds-en-ligne.ird.fr/foret].

Dounias E., Lehébel-Péron A. \& Schatz B. 2011 - L'abeille noire, le châtaignier et l'homme dans les Cévennes : une suave alliance en forme de ruche-tronc. In : Pietrasanta Y. \& Schatz B. (Ed.) Le génie de la nature. Mèze, Biotope édition : 70-85.

Duby G. 1970 - L'économie rurale et la vie des campagnes dans l'occident médiéval (France, Angleterre, Empire, IX $\mathrm{X}^{e}-\mathrm{XV} \mathrm{V}^{e}$ siècles). Paris, Aubier.

Egan D. \& Howell E.A. 2005 - The Historical Ecology Handbook: A Restorationist's Guide to Reference Ecosystems. Washington DC., Island Press.

Engel M.S. 1999 - The taxonomy of recent and fossil honey bees (Hymenoptera: Apida: Apis). J.

Hymenopt. Res. 8 : 165-196.

Faral E. 1948 - La vie quotidienne au temps de Saint Louis. Hachette.

Fernández-Salas A., Alonso-Díaz M.A., Acosta-Rodríguez R., Torres-Acosta J.F.J., Sandoval-Castro C.A. \& Rodríguez-Vivas R.I. 2011 - In vitro acaricidal effect of tanin-rich plants against the cattle tick Rhipicephalus (Boophilus) microplus (Acari: Ixodidae). Vet Parasitol 175 (1) : 113-118.

Food \& Agriculture Organization of the United Nations, 2009 - FAOSTAT.

Franck P., Garnery L., Celebrano G., Solignac M. \& Cornuet J.-M. 2000 - Hybrid origins of honeybees from Italy (Apis mellifera ligustica) and Sicily (A. m. sicula). Mol. Ecol. 9 : 907-921.

Gallai N., Salles J.M., Settele J. \& Vaissière B.E. 2009 - Economic valuation of the vulnerability of world agriculture confronted with pollinator decline. Ecological economics $68: 810-821$. 
Galzin J. 1986 - Déboisement et plantation de châtaignier en Cévennes. Annales du Parc National des Cévennes. Florac.

Garnery L., Franck P., Baudry E., Vautrin D., Cornuet J.-M. \& Solignac M. 1998 - Genetic diversity of the west European honey bee (Apis mellifera mellifera and A. m. iberica) I. Mitochondrial DNA. Genet. Sel. Evol. 30 : 31-47.

Garnery L., Vautrin D., Cornuet J.-M. \& Solignac M. 1991 - Phylogenetic relationships in the genus Apis inferred from mitochondrial DNA sequence data. Apidologie 22 : 87-92.

IPBES 2016 - Summary for policymakers of the assessment report of the Intergovernmental Science-Policy Platform on Biodiversity and Ecosystem Services on pollinators, pollination and food production. Potts S.G., Imperatriz-Fonseca V.L., Ngo H.T., Biesmeijer J.C., Breeze T.D., Dicks L.V., Garibaldi L.A., Hill R., Settele J. \& Vanbergen A.J. (Ed.) : 1-30.

Jensen A.B., Palmer K.A., Boomsma J.J., \& Pedersen B.V. 2005 - Varying degrees of Apis mellifera ligustica introgression in protected populations of the black honeybee, Apis mellifera mellifera, in northwest Europe. Mol. Ecol. 14 : 93-106.

Jobard E. 2012 - Mise en place d'un observatoire de l'apiculture au sein du Parc National des Cévennes. Rapport de licence, $39 \mathrm{p}$.

Layens G. de \& Bonnier G. 1898 - Cours complet d'apiculture (culture des abeilles). Paris, P. Dupont.

Lehébel-Péron A. 2014 - L'abeille noire et la ruche-tronc. Approche pluridisciplinaire de l'apiculture traditionnelle cévenole : histoire, diversité et enjeux de conservation. Thèse de l'Université de Montpellier II, $227 \mathrm{p}$.

Lehébel-Péron A., Sidawy P., Dounias E. \& Schatz B. 2016 - Attuning local and scientific knowledge in the context of global change: The case of heather honey production in southern France. Journal of Rural Studies $44: 132-142$.

Lehébel-Péron A., Sidawy P., Dounias E. \& Schatz B. 2015 - L'apiculture traditionnelle, témoin des changements de paysages en Cévennes. In Maire E. \& Laffly D. (Ed.) Abeilles et paysages, Quæ : 159-172.

Liger L. \& Bernier H. 1775 - La nouvelle maison rustique, ou Économie générale de tous les biens de campagne: la manière de les entretenir \& de les multiplier. Éditions Samson.

Maire E. \& Laffly D. 2015 - Abeilles et paysages. Quæ.

Marchenay P. 1979 - L'homme et l'abeille. Berger-Levrault.

Noel P. 1893 - L'élevage des abeilles par l'emploi de la nouvelle ruche à cadres mobiles, Bulletin de la Société d'Agriculture Industrie, Sciences et Art du département de la Lozère. Mende.

Oldroyd B.P. 2007 - What's killing American honey bees? PLoS Biol. 5 : e168.

Parc national des Cévennes 2007 - Atlas archéologique du Parc national des Cévennes.

Perrier C., Strange J., Langella O., Sheppard S.W. \& Garnery L. 2003 - Diversité génétique, introgressions mitocondriales et nucléaires dans une population d'abeilles des Landes de Gascogne. Actes Bur. Ressour. Génétiques 4 : 79-100.

Redon J. 1977 - Géographie humaine préhistorique du Bassin des Gardons au Paléolithique (Thèse de doctorat : Archéologie préhistorique). Université de Toulouse Le Mirail.

Riondet J. 2013 - Le rucher durable : Guide pratique de l'apiculteur d'aujourd'hui. Les Editions Eugen Ulmer. 
Robert P., Rey A., Rey-Debove J., 2006 - Le Nouveau Petit Robert: dictionnaire alphabétique et analogique de la langue française. Dictionnaires Le Robert.

Roffet-Salque M., Regert M., Evershed R. P., Outram A. K., Cramp L. J. E., Decavallas O., Dunne J., Gerbault P., Mileto S., Mirabaud S., Pääkkönen M., Smyth J., Šoberl L., Whelton H.L., Alday-Ruiz A ., Asplund H., Bartkowiak M., Bayer-Niemeier E., Belhouchet L., Bernardini F., Budja M., Cooney G ., Cubas M., Danaher E.M., Diniz M., Domboróczki L., Fabbri C., González-Urquijo J.E., Guilaine J., Hachi S., Hartwell B.N., Hofmann D., Hohle I., Ibáñez J.J., Karul N., Kherbouche F., Kiely J., Kotsakis K., Lueth F., Mallory J.P., Manen C., Marciniak A., Maurice-Chabard B., Mc Gonigle M.A., Mulazzani S., Özdoğan M., Perić O.S., Perić S.R., Petrasch J., Pétrequin A.M., Pétrequin P., Poensgen U., Pollard C.J. Poplin F., Radi G., Stadler P., Stäuble H., Tasić N., Urem-Kotsou D., Vuković J.B., Walsh F., Whittle A., Wolfram S., Zapata-Peña L. \& Zoughlami J. 2015 - Widespread Exploitation of the Honeybee by Early Neolithic Farmers. Nature $527: 226-30$. DOI:10.1038/ nature15757.

Rortais A., Arnold G., Alburaki M., Legout H. \& Garnery L. 2010 - Review of the DraI COICOII test for the conservation of the black honeybee (Apis mellifera mellifera). Conserv. Genet. Resour. 3 : 383-391. doi:10.1007/s12686-010-9351-x

Roué M., Battesti V., Césard N. \& Simenel R. 2015 - Ethnoecology of pollination and pollinators. Revue d'Ethnoécologie 7. DOI: 10:4000/ethnoécologie.2229.

Ruttner F. 1988 - Biogeography and taxonomy of honeybees. Springer-Verlag.

Serres O. (de) 1600 - Le théâtre d'agriculture et mesnage des champs, d' Olivier de Serres, seigneur du Pradel, dans lequel est représenté tout ce qui est requis et nécessaire pour bien dresser, gouverner, enrichir et embellir la maison rustique. Paris, Éditions Jamet Métayer.

Sheppard W.S. \& Berlocher S.H. 1989 - Allozyme variation and differentiation among four Apis species. Apidologie $20: 419-431$.

Sheppard W.S. \& Meixner M.D. 2003 - Apis mellifera pomonella, a new honey bee subspecies from Central Asia. Apidologie 34 : 367-375. doi:10.1051/apido:2003037

Strange J.P., Garnery L. \& Sheppard W.S. 2007 - Persistence of the Landes ecotype of Apis mellifera mellifera in southwest France: confirmation of a locally adaptive annual brood cycle trait.

Apidologie 38 : 259-267.

Swetnam T.W., Allen C.D. \& Betancourt J.L. 1999 - Applied historical ecology: using the past to manage for the future. Ecological Applications 9 (4): 1189-1206.

Travier D. 2006 - Que sait-on de l'histoire de la châtaigneraie cévenole ? In Chassany J.-P; \& Crosnier C. (Ed.) Le Renouveau de la châtaigneraie cévenole : 304.

Vanengelsdorp D. \& Meixner M.D. 2010 - A historical review of managed honey bee populations in Europe and the United States and the factors that may affect them. J. Invertebr. Pathol. 103 : 80-95.

Williams I.H. 1994 - The dependence of crop production within the European Union on pollination by honey bees. Agricultural Zoology Reviews $6: 229-257$.

\section{NOTES}

1. La ruche à cadres telle qu'elle est connue aujourd'hui semble avoir été inventée dans les années 1850 simultanément en divers pays d'Europe et aux États-Unis. Français immigré aux USA en 1863, Charles Dadant décrit sa ruche à cadres mobiles dans un journal français et publie un 
livre d'apiculture en français, en 1874. Le mot « apiculteur » est né en 1845 (Le Robert et al. 2006). Il serait donc quasiment contemporain de l'invention des premières ruches à cadres mobiles. Ce pourrait être, au niveau du vocabulaire, une reconnaissance de la créativité des éleveurs d'abeilles, dans la recherche et la mise en œuvre de nouvelles techniques.

2. Nos études en cours semblent montrer une faible charge en agents pathogènes dans les ruches-troncs, qui pourrait être imputée à la teneur élevée en tanins du bois de châtaignier.

\section{RÉSUMÉS}

L'unicité de l'abeille domestique sur toute l'Europe sous-entend à tort une relative homogénéité continentale des pratiques apicoles, au point de négliger la compréhension de l'évolution de cette activité à un niveau plus strictement local. Cette connaissance est pourtant essentielle pour concevoir des modes satisfaisants de gestion locale des ressources dans des systèmes socioécologiques profondément marqués par les activités apicoles. Une étude d'ethnoécologie historique a été conduite sur les savoirs locaux en matière d'apiculture dans le Parc national des Cévennes (Sud de la France). Combinant l'exploitation de documents d'archives, de littérature scientifique et de témoignages obtenus auprès de Cévenols âgés, cette plongée dans l'histoire a permis de reconstituer les épisodes marquants de l'activité apicole en Cévennes, à travers les modifications de types de ruches et de races d'abeilles employés, et de valorisation des produits de la ruche au gré des opportunités sociales et économiques successives. Les éléments les plus saillants, telle que la preuve formelle de l'exploitation de ruches-troncs au début du XVII ${ }^{\mathrm{e}}$ siècle, ont ainsi pu être datés et rétrospectivement mis en contexte. L'élevage artisanal d'une abeille noire locale en ruche-tronc s'est maintenu jusque dans les années 1970 avant un basculement dans une apiculture en ruche à cadres s'accompagnant d'une adoption de nouvelles races d'abeilles et d'une professionnalisation de la filière. Les apiculteurs cévenols sont donc tardivement passés d'une apiculture domestique et paysagère, optimisée dans un contexte de pluriactivité de subsistance, à une apiculture de rente en quête de maximisation de la production de miel, associée à une diversification des races d'abeilles et à leur hybridation. Cette mise en perspective historique et bioculturelle de l'apiculture cévenole permet d'ajuster des objectifs raisonnables de conservation afin de tenter une conciliation entre la valeur patrimoniale d'une apiculture traditionnelle et le renforcement d'une filière économique en devenir.

On the grounds that beekeeping of the single domesticated honeybee looks at first sight homogenous throughout whole Europe, understanding the evolution of this activity at the very local level is generally overlooked. Such understanding is nonetheless crucial to conceive appropriate community-based resource management in socioecological systems that have been profoundly imprinted by beekeeping activities. We carried out an ethnoecological history study focusing on local knowledge regarding beekeeping in the Cevennes National Park (southern France). By combining the analysis of archival documents, scientific literature and personal testimonies from elderly Cevennes dwellers, this dive into history has allowed us to reconstitute the major episodes of beekeeping in Cevennes, by considering the modifications of chosen beehive models and bee landraces, as well as the valorization of beehive products following the evolving social and economic circumstances. Most salient features, such as the first evidence of the use of log hives in the early 17th century, were thus time-stamped and retrospectively set into context. Artisanal beekeeping of the local black bee hosted in log hives has persisted until 
the 1970s date of the transition to modern beekeeping using frame hives, selected bee landraces, and a professionalization of the local honey trade sector. Beekeepers from the Cevennes region only lately stepped from a domestic and landscaped beekeeping, which was optimized in a context of self-sufficient pluriactivity, into an intensive beekeeping driven by the search for maximized honey yields and supported by a diversification and a hybridization of bee landraces. Such combined historical and biocultural perspectives of beekeeping in Cevennes should serve to elaborate reasonable goals for conservation and should help conciliating the preservation of a patrimonial and traditional beekeeping along with the enhancement of a yet emerging local honeybee market.

INDEX

Keywords : historical ethnoecology, Cevennes, beekeeping, log hives, local knowledge Index géographique : Cévennes

Mots-clés : ethnoécologie historique, apiculture, ruche-tronc, savoirs locaux

\section{AUTEURS}

\section{AMELINE LEHÉBEL-PÉRON}

Centre d'Écologie Fonctionnelle et Évolutive (CEFE) UMR 5175, CNRS - Université de Montpellier Université Paul-Valéry Montpellier - EPHE - IRD, 1919 route de Mende, 34293 Montpellier Cedex 5 France

Parc National des Cévennes, BP 15 Le Château, 48400 Florac

\section{DANIEL TRAVIER}

Musée des vallées cévenoles, BP 8, 30270 St-Jean-du-Gard

\section{ALAIN RENAUX}

Le Courtinaou, 30460 Sainte-Croix-de-Caderle

\section{EDMOND DOUNIAS}

Centre d'Écologie Fonctionnelle et Évolutive (CEFE) UMR 5175, CNRS - Université de Montpellier Université Paul-Valéry Montpellier - EPHE - IRD, 1919 route de Mende, 34293 Montpellier Cedex 5 France

\section{BERTRAND SCHATZ}

Centre d'Écologie Fonctionnelle et Évolutive (CEFE) UMR 5175, CNRS - Université de Montpellier Université Paul-Valéry Montpellier - EPHE - IRD, 1919 route de Mende, 34293 Montpellier Cedex 5 France

bertrand.schatz@cefe.cnrs.fr 